\title{
Canker and Wood Rot Pathogens Present in Young Apple Trees and Propagation Material in the Western Cape of South Africa
}

\author{
Minette Havenga, ${ }^{1}$ Greg M. Gatsi, ${ }^{1}$ Francois Halleen, ${ }^{1,2}$ Christoffel F. J. Spies, ${ }^{1,2}$ Rhona van der Merwe, ${ }^{1}$ and Lizel Mostert ${ }^{1, \dagger}$ \\ ${ }^{1}$ Department of Plant Pathology, University of Stellenbosch, Private Bag X1 Matieland, 7602, South Africa \\ ${ }^{2}$ Plant Protection Division, ARC Infruitec-Nietvoorbji, Private Bag X5026, Stellenbosch, 7599, South Africa
}

\begin{abstract}
Canker and wood rot pathogens cause dieback and, in severe cases, the death of young apple trees. Recently, a higher occurrence of cankers was observed on 1-year-old apple trees in the Western Cape Province of South Africa. This study aimed to assess the phytosanitary status of nursery trees and propagation material as possible inoculum sources for canker pathogens. Thirteen 1-year-old apple orchards showing canker or dieback symptoms were sampled. Certified nursery apple trees were collected from four nurseries as well as scion and rootstock mother plant material. Isolations were made from the discoloration observed in the vascular tissue of the plant parts and from asymptomatic material. Possible canker and wood rot species were identified with PCR and sequence comparisons of the relevant gene regions and phylogenetic analyses. Similar canker and wood rot species were isolated from 1-year-old diseased apple trees, nursery apple trees, and the propagation material.

disease on commercial 1-year-old trees were also found in high numbers causing latent infection in certified apple nursery trees. These species were Didymosphaeria rubi-ulmifolii sensu lato, Diplodia seriata, Schizophyllum commune, Didymella pomorum, and Coniochaeta fasciculata, with $D$. rubi-ulmifolii sensu lato being the dominant species in both sampling materials. In all, $65 \%$ of certified nursery apple trees, $5 \%$ of scion shoots used for budding, and $21 \%$ of rooted rootstock cuttings from layer blocks had latent infections of canker and wood rot pathogens. Pathogenicity trials were conducted with isolates of 39 species, inoculated onto 2 year-old branches of 14-year-old Golden Delicious trees. All species caused lesions that were significantly longer than the control. This study confirmed the presence of canker and wood rot pathogens in apple propagation material as well as certified nursery apple trees, which will aid the improvement of management practices in nurseries.
\end{abstract} Forty-five fungal species associated with canker or wood rot symptoms were identified. The top five most abundant fungal species found causing
Keywords: canker, dieback, Malus spp., nursery, wood rot
Dieback of apple trees is caused by both true and opportunistic canker and wood rot pathogens giving rise to general symptoms such as cankers, twig blight, and wood rotting (Borovinova et al. 2012; Brown-Rytlewski and McManus 2000; Menapace et al. 2015). The pathogens responsible for canker and wood rot symptoms infect their hosts through wounds and colonize the vascular tissue, resulting in blockage of the vascular system (Cloete et al. 2011). Infected trunks, branches, and shoots start dying back, eventually leading to the death of the entire tree or even failure of the orchard (Zhang et al. 2014). The farmer can face major financial damages when the infections are severe enough for the orchards to become uneconomical because dieback reduces the yield and longevity of the host (Elfar et al. 2013).

Fifteen canker pathogens have been reported to cause dieback or canker symptoms on apple trees in South Africa. These include Botryosphaeria dothidea (Moug.) Ces. \& De Not., Neofusicoccum ribis (Slippers, Crous \& M. J. Wingf.) Crous, Slippers \& A. J. L. Phillips, Diplodia mutila (Fr.) Mont., D. seriata De Not., N. australe (Slippers, Crous \& M. J. Wingf.) Crous, Slippers \& A. J. L. Phillips, Cryptosporiopsis corticola (Edgerton) Nannf, Cytospora schulzeri

Current address of C. F. J. Spies: ARC-Plant Health and Protection, Private Bag X5017, Stellenbsoch, 7599, South Africa.

${ }^{\dagger}$ Corresponding author: L. Mostert; 1most@sun.ac.za

Funding: We thank Hortgro Science, the National Research Foundation (grant numbers RDYR14080285149 and TP14080888887), and the Technology and Human Resources for Industry Programme for funding this project.

*The $\boldsymbol{e}$-Xtra logo stands for "electronic extra" and indicates that 19 supplementary figures and four supplementary tables are published online.

The author(s) declare no conflict of interest.

Accepted for publication 26 June 2019.

(C) 2019 The American Phytopathological Society
Sacc. \& P. Syd., Diaporthe ambigua Nitschke, D. foeniculina (Sacc.) Udayanga \& Castl., C. leucostoma (Pers.) Sacc., Cytospora nivea (Hoffm.) Sacc., species of the family Didymellaceae, Phaeoacremonium fraxinopennsylvanicum (T. E. Hinds) Gramaje, L. Mostert \& Crous, and P. minimum (Tul. \& C. Tul.) Gramaje, L. Mostert \& Crous (Adams et al. 2006; Cloete et al. 2011; Crous et al. 2000; Smit et al. 1996; Smit et al. 1997; Sutton et al. 2015). Four Basidiomycetes species are associated with wood rot symptoms on apple trees in South Africa. These include Bjerkandera adusta (Willd.) P. Karst., Chondrostereum purpureum (Pers.) Pouzar, Schizophyllum commune Fr., and Trametes versicolor (L.) Lloyd (Crous et al. 2000; Matthee and Thomas 1977; Sutton et al. 2015).

Canker and wood rot symptoms are commonly observed in older apple orchards in South Africa. The decline and eventual death of these trees can take an extended period of time (Smit et al. 1996). In contrast, younger trees infected with canker or wood rot pathogens can die more rapidly, especially when exposed to abiotic stress factors (Marek et al. 2013; Smit et al. 1996). Studies conducted in Europe on the death of young, newly established apple trees found that young trees developed large cankers on the main stems shortly after establishment due to infections by Cylindrocarpon heteronema (Berk. \& Broome) Wollenw. (Brown et al. 1994) and Neonectria ditissima Tul. \& C. Tul. Samuels \& Rossman (McCracken et al. 2003). Both of these studies concluded that the cankers observed in the young apple orchards originated from latent infections that occurred during the propagation process in the nursery.

Marek et al. (2013) investigated the causal agents of cankers forming on young apple trees that were held in cold storage. The study found that latent infections in nursery trees caused disease symptoms during cold storage or shortly after planting (Marek et al. 2013). A study done by Fujita et al. (1988) found that mature apple trees artificially inoculated with the canker pathogen Diaporthe tanakae Tak. Kobay. \& Sakuma only exhibited typical canker lesions after 2 years. The time required for symptom development of canker and wood rot together with stress due to cold storage used at producer levels explains the absence of symptoms in the nursery. 
Recently, a higher occurrence of canker development was observed on 1-year-old trees in the same season of establishment in the Western Cape Province of South Africa. Two incidences of serious losses (30 and 70\%) of newly planted apple trees due to stem cankers have been reported (Disease Clinic, Department of Plant Pathology, Stellenbosch University). Over the past 6 years, 60 trees between the ages of 1 and 3 years old were sent to the Disease Clinic of Stellenbosch University for analysis. These 60 trees represented 60 commercial orchards in the Western Cape with canker or wood rot symptoms. The deciduous fruit industry of South Africa launched an investigation into the reasons behind observed losses of 1-year-old apple trees of specific rootstocks.

Knowledge regarding latent infections of canker or wood rot pathogens in certified nursery trees in South Africa is lacking. The aim of this study was to assess the phytosanitary status of nursery apple trees and propagation material. The objectives of this study were to (i) determine the occurrence of canker and wood rot pathogens in 1-yearold apple trees showing cankers or dieback, certified nursery apple trees, and plant material used during the propagation of apple trees and (ii) assess the pathogenicity of the isolated fungi.

\section{Materials and Methods}

Sampling of planting material. One-year-old orchards. Thirteen 1 -year-old commercial orchards in the Kouebokkeveld, Hermanus, and Grabouw areas were surveyed in March 2015 and March 2016. These orchards exhibited dieback symptoms within 1 year of establishment. Ten trees exhibiting canker symptoms were sampled per orchard. The trees were removed with their roots to ensure that the dieback symptoms were not due to apple replant. In total, 130 trees were sampled over the 2-year period.

Certified nursery apple trees. Nursery plants with scion Golden Delicious and rootstocks MM109, M793, and Geneva (G222) were collected from four fruit tree nurseries in South Africa. In total, 480 trees (160 trees per rootstock) were collected in August to September 2015. Due to the unavailability of some scion-rootstock combinations, other scion cultivar options were also accepted when the Golden Delicious cultivar was not available. Forty trees with the rootstock CG4202 were also collected instead of G222 rootstock, forming part of the Geneva rootstock sampling. Trees belonging to different size classes were also requested from these nurseries (Supplementary Table S1).

Scion mother block orchards. Scion mother block trees were sampled in February and March 2015 and 2016. Three scion cultivars were used, including Rosy Glow, Golden Delicious, and Early Red One. In all, 6 scion mother blocks per scion cultivar were sampled, which resulted in 18 mother blocks in total, and 15 (2016) or 20 (2015) trees were sampled per block. The first year's results indicated that 15 trees would be adequate, and the number was therefore reduced for the following year's sampling. From each tree, a typical canker symptom and pruning wound, as well as a 1-year-old shoot, were sampled. If there were no cankers present on the tree, two pruning wounds were sampled, and if there were more than one canker present on the tree, two cankers were sampled (Supplementary Table S2).

Rootstock mother blocks. Rootstock plant material was collected in June 2016 in collaboration with the three Plant Improvement Organizations (PIOs) of South Africa. Three rootstock cultivars were selected; namely, G222, M793, and MM109. Three layer blocks per rootstock were investigated, and 45 asymptomatic shoots were randomly removed from each block. In total, 405 asymptomatic rooted rootstocks were collected. In one of the nurseries, dieback symptoms were found in the MM109 and M793 1-year-old nursery blocks. These are the rootstock cuttings, which were removed from the layer blocks and established in the nursery soil in August 2015. Dieback occurred on the rootstock shoots shortly after establishment. In all, 45 M793 and 42 MM109 symptomatic shoots were sampled from the corresponding 1-year-old nursery blocks.

Isolation from planting material. One-year-old commercial apple orchards. Canker lesions were excised from the tree and triple surface sterilized by soaking in $70 \%$ ethanol solution for $30 \mathrm{~s}$, then
$1 \% \mathrm{NaOCl}$ solution for $60 \mathrm{~s}$, and, finally, in $70 \%$ ethanol solution for $30 \mathrm{~s}$. The cankered wood pieces were left to air dry on sterile tissue paper in the laminar flow cabinet. A pruning shear (with blades flame sterilized) was used to cut the wood pieces in half lengthwise, exposing the vascular tissue. Twelve wood pieces measuring approximately 2 by $2 \mathrm{~mm}$ were removed from the section between the diseased dark-brown vascular discoloration and the healthy tissue, and placed onto $2 \%$ potato dextrose agar (Biolab, Midrand) amended with streptomycin sulfate (PDA+s; $40 \mathrm{mg}$ /liter; Calbiochem, Merck). Dishes were incubated at $23^{\circ} \mathrm{C}$ under natural light for 2 to 3 weeks, or until substantial fungal growth was observed. Representative subcultures were made, using the hyphal tip method, from each primary isolation dish and incubated under the same conditions. Preparation of plant material and isolations were done throughout the study as described in the sampling of 1-year-old tissues.

Certified nursery apple trees. Isolations were made from four parts found on the nursery trees; namely, wounds made on the scion shoot, the bud union, the pruning wound made when the rootstock was cut back, and additional wounds found on the rootstock. If no wounds were found on the scion or rootstock shoot, asymptomatic pieces were used.

Scion mother blocks orchards. Isolations were made from the vascular discoloration found in the cankers and pruning wounds. Furthermore, eight buds were removed from each scion shoot and placed onto PDA+s. Four disks were also cut through the internodes of the scion shoot and placed onto PDA+s.

Rootstock mother blocks. Isolations were made from asymptomatic tissue. Disks were cut though the buds on the rootstock shoot of the rooted cutting with a sterile pruning shear and placed on PDA+s. Disks were cut into quarters and placed onto PDA+s. Three disks were removed from each rooted cutting and placed onto three PDA+s dishes. Isolations were made from the internal vascular discoloration of the 1-year-old nursery material and fruiting bodies on the canker were scraped off and placed onto PDA+s.

Identification of fungal species. Fungal isolates were arranged into different morphological groups based on cultural and morphological characteristics. Cultural characteristics included colony size, color, texture, and shape. Microscopic slides were made from unknown cultures to observe conidia shape and color. Microscopic slides were examined with a Nikon Eclipse E600 compound microscope. Due to the high diversity of fungal genera, representatives of cultural and morphological groups were selected for further molecular identification. All known saprophytes (e.g., Fusarium, Alternaria, Epicoccum, and Aureobasidium spp., among others) were discarded. One isolate per morphological group for each plant part was stored and used for further identification.

\section{Molecular identification.}

DNA extraction, PCR, and sequencing. Ten isolates from each morphological group were selected for molecular identification. DNA was extracted from 3 week-old fungal cultures on PDA using the protocol of Damm et al. (2008). Primer pairs were selected according to the taxonomic groups.

For species of Diatrypaceae, Diaporthales, Basidiomycetes, and unidentified fungal cultures, the internal transcribe spacers (ITS) 1 and 2 and the 5.8S ribosomal DNA gene area were amplified with ITS5 and ITS4 (White et al. 1990). In a total reaction volume of $20 \mu \mathrm{l}$, the PCR contained $1 \mu \mathrm{l}$ of DNA, $1 \times$ KAPA Taq ready mix (KAPABiosystems), $0.4 \mu \mathrm{M}$ each primer, and double-distilled (dd) $\mathrm{H}_{2} \mathrm{O}$. Reaction conditions consisted of an initial denaturation step at $94^{\circ} \mathrm{C}$ for $5 \mathrm{~min}$; followed by 30 cycles of $30 \mathrm{~s}$ at $94^{\circ} \mathrm{C}, 30 \mathrm{~s}$ at $55^{\circ} \mathrm{C}$, and $30 \mathrm{~s}$ at $72^{\circ} \mathrm{C}$; and a final extension step at $72^{\circ} \mathrm{C}$ for $7 \mathrm{~min}$.

For species in the Botryosphaeriaceae family and Diaporthales order, the elongation factor 1- $\alpha$ gene $(E F 1-\alpha)$ was amplified with primers EF1-728F and EF1-968R (Carbone and Kohn 1999) following the previously described PCR volumes. PCR conditions consisted of an initial denaturation step at $94^{\circ} \mathrm{C}$ for $5 \mathrm{~min}$; followed by 30 cycles of $45 \mathrm{~s}$ at $94^{\circ} \mathrm{C}, 45 \mathrm{~s}$ at $53^{\circ} \mathrm{C}$, and $90 \mathrm{~s}$ at $72^{\circ} \mathrm{C}$; with a final extension step at $72^{\circ} \mathrm{C}$ for $7 \mathrm{~min}$. Fungal cultures with $D$. seriata-like morphological characteristics were identified using the species-specific primers DS3.8 S3 and DS3.8 R6 (Martín et al. 2014). In a total reaction volume of $20 \mu \mathrm{l}$, the PCR contained $1 \mu \mathrm{l}$ of genomic 
DNA, $8 \mu \mathrm{l}$ of $2 \times$ KAPA Taq ready mix, $0.8 \mu \mathrm{l}$ of ITS-5 (0.4 pmol/ $\mu \mathrm{l}), 0.8 \mu \mathrm{l}$ of ITS-4 $(0.4 \mathrm{pmol} / \mu \mathrm{l})$, and $8.4 \mu \mathrm{l}$ of double-distilled $\mathrm{H}_{2} \mathrm{O}$. Reaction conditions consisted of an initial denaturation step at $95^{\circ} \mathrm{C}$ for $5 \mathrm{~min}$; followed by 35 cycles of $30 \mathrm{~s}$ at $94^{\circ} \mathrm{C}, 45 \mathrm{~s}$ at $57^{\circ} \mathrm{C}$, and $45 \mathrm{~s}$ at $72^{\circ} \mathrm{C}$; with a final extension step at $72^{\circ} \mathrm{C}$ for $7 \mathrm{~min}$.

Species of Phaeoacremonium and Diatrypaceae were identified with the partial $\beta$-tubulin gene amplified using the primers T1 (O'Donnell and Cigelnik 1997) and Bt2B (Glass and Donaldson 1995). The same PCR volumes and concentrations were used as described earlier. Reaction conditions consisted of an initial denaturation step at $94^{\circ} \mathrm{C}$ for $5 \mathrm{~min}$; followed by 36 cycles of $45 \mathrm{~s}$ at $94^{\circ} \mathrm{C}, 45 \mathrm{~s}$ at $55^{\circ} \mathrm{C}$, and $90 \mathrm{~s}$ at $72^{\circ} \mathrm{C}$; with a final extension step at $72^{\circ} \mathrm{C}$ for 6 min. All PCRs were performed in an Applied Biosystems 2700 PCR machine. A nontemplate control was also included in each PCR run. PCR products were separated by electrophoresis on a $1 \%(\mathrm{wt} / \mathrm{vol})$ agarose gel in Tris-acetateEDTA running buffer (0.4 M Tris, $0.05 \mathrm{M}$ NaAc, and 0.01 M EDTA, $\mathrm{pH}$ 7.5) after ethidium bromide staining. The GeneGenius Gel Documentation and Analysis System (Syngene) were used to visualize the gel under UV light alongside a 100-bp DNA ladder (GeneRuler; Thermo Fisher Scientific).

PCR products were purified using the MSB Spin PCRapase kit (Invitek) and prepared for forward and reverse sequencing. Thermocycler conditions were $1 \mathrm{~min}$ at $95^{\circ} \mathrm{C} ; 30$ cycles of $10 \mathrm{~s}$ at $95^{\circ} \mathrm{C}, 5 \mathrm{~s}$ at $50^{\circ} \mathrm{C}$, and $4 \mathrm{~min}$ at $60^{\circ} \mathrm{C}$; with a final extension of $30 \mathrm{~s}$ at $60^{\circ} \mathrm{C}$. The gene areas were sequenced using ABI PRISM Big Dye Terminator v3.1 Cycle Sequencing Ready Reaction Kit (PE Biosystems) with the primers used in the initial PCRs. The nucleotide order of samples was read in an ABI 3130xl DNA sequencer (Perkin-Elmer) at the DNA Sequencing Unit at the Central Analytical Facility of Stellenbosch University.

Phylogenetic analyses. Forward and reverse sequences for each isolate were aligned in Geneious R 9.1.7 (Biomatters Ltd.) and a consensus sequence was extracted. A Basic Local Alignment Search Tool (BLAST) search was conducted against the NCBI (https:// www.ncbi.nlm.nih.gov/) nucleotide database. Isolates obtained from this study were aligned with representative sequences from each taxonomic group obtained from GenBank (https://www.ncbi.nlm.nih.gov/ Genbank) in Geneious R 9.1.7. Individual taxonomic group sequences' data sets were aligned with L-INS-I method using the program MAFFT v7.222 (Katoh and Standley 2013; Katoh et al. 2002) in Geneious R 9.1.7. Maximum-likelihood (ML) analyses were performed using PhyML (Guindon and Gascuael 2003; Guindon et al. 2010) in Geneious R 9.1.7 under the general time-reversible model. Both the $\gamma$ distribution parameter and proportion of invariable sites were estimated. Bootstrap support values were calculated from 1,000 replicates. Clades with bootstrap support $\geq 70 \%$ were considered significant and highly supported (Hillis and Bull 1993).

Pathogenicity trial. Seventy-two fungal isolates representing 39 species collected and identified from young apple trees were used in the pathogenicity trial. The isolates were placed on PDA+s and incubated at $25^{\circ} \mathrm{C}$ under natural light. Pathogenicity studies were conducted on 14-year-old Golden Delicious apple trees in Grabouw. Two-year-old healthy shoots were surface sterilized with $70 \%$ ethanol. The bark was removed with a sterilized 4-mm cork borer, whereafter a hole of 2 to $2.5 \mathrm{~mm}$ deep was drilled using a sterilized 4-mm drill bit. A mycelium plug was placed into the wound and sealed with Parafilm. A PDA+s plug was used as a control. Inoculations were made at least $10 \mathrm{~cm}$ from the main branch. Ten shoots were used per tree. The trial layout was a balanced incomplete block design with 73 treatments (including the PDA+s as the control). Each tree was seen as an incomplete block and each treatment was replicated 10 times. The trial was repeated on another 14-year-old Golden Delicious orchard on the same farm. Shoots were harvested after 5 months. Isolations were conducted in the same way as described earlier. The identity of the isolates used in the pathogenicity tests were confirmed by sequencing the relevant barcoding gene region for one isolate per species.

Statistical analyses. To test for differences in the frequencies of the different factors, $\chi^{2}$ tests were performed using Frequency Procedure (PROC FREQ) of the SAS software, version 9.2 (SAS
Institute Inc.). The percentage of infected trees was calculated. Factor (treatment) means (i.e., PIO, cultivars, rootstocks etc.) were tested using PROC TTEST with SAS software. The number of infections caused by the different pathogens was also expressed as a percentage of the total number of infected trees for the different factors (treatments). Normality of standardized residuals was confirmed by the Shapiro-Wilk test (Shapiro and Wilk 1965). Levene's test was used to verify the homogeneity of factor (treatment) variances (Levene 1960). The data were subjected to analysis of variance (ANOVA) using the General Linear Models Procedure (PROC GLM) of SAS software. Fisher's least significant difference (LSD) was calculated at the 5\% level to compare factor (treatment) means (Ott and Longnecker 2001).

For the pathogenicity trials, the data (lesion length and fungal reisolation percentages) from the two trials were subjected to ANOVA separately according to the experimental design, using PROC GLM of SAS statistical software (version 9.4; SAS Institute Inc.). After testing whether trials were of comparable precision by means of Levene's test for homogeneity of variance (Levene 1960), trial results were combined and investigated in one overall ANOVA, as described by John and Quenouille (1977). Where the requirement of homogeneity of trial variance was not met, a weighted ANOVA (weighing with trial variances) was performed. The Shapiro-Wilk test (W test) was performed to test for normality (Shapiro and Wilk 1965). Lesion length was $\operatorname{Ln}(x+1)$ transformed to improve normality (Snedecor and Cochran 1980). Student's $t$ LSD was calculated at the $5 \%$ level to compare treatment means. A probability level of 5\% was considered significant for all tests.

\section{Results}

Diversity of fungal taxa. In total, 45 fungal species belonging to the genera Bjerkandera, Botryosphaeria, Cadophora, Chondrostereum, Coniochaeta, Cytospora, Diaporthe, Didymella, Didymosphaeria, Diplodia, Eutypa, Neofusicoccum, Peniophora, Phaeoacremonium, Schizophyllum, Stereum, Trametes, and Truncatella were identified in this study (Table 1; Supplementary Figs. 1, 2, 3, 4, 5, 6, 7, 8, 9, 10, 11, $12,13,14$, and 15). The majority of the species $(n=38)$ are species of Ascomycetes and only seven species belong to the division Basidiomycetes. Didymosphaeria rubi-ulmifolii sensu lato was the predominant fungal species isolated from 276 plant parts throughout the study, and was the predominant pathogen found in the 1-year-old commercial trees (present in 11 of the orchards investigated) as well as the nursery material (241 plant parts) (Table 2). Five pathogens were found in all four sampled plant material types; namely, S. commune, D. seriata, D. foeniculina, Didymosphaeria variabile, and D. rubi-ulmifolii sensu lato. Apart from the possible canker or wood rot species, a variety of saprophytic species from genera such as Trichoderma, Epicoccum, Alternaria, Aureobasidium, and Penicillium were also isolated.

One-year-old commercial apple orchards. From the 130 diseased 1-year-old trees, possible canker and wood rot pathogens were found in 55 trees (42.3\%). Twenty-one different fungal taxa were isolated from dieback symptoms. The most predominant pathogen was $D$. rubi-ulmifolii sensu lato, isolated from 15 trees, followed by $D$. seriata (isolated from 11 trees) and Eutypella citricola (isolated from 5 trees) (Table 2). Typical symptoms observed on the 1-year-old trees were constricting cankers (Fig. 1A). A cross section through the cankers exposed dark-brown vascular discoloration, streaking down into the live tissue of the tree (Fig. 1B, C). The majority of trees sampled had welldeveloped root systems, indicating that the aboveground symptoms were not due to soilborne diseases. Cankers were found on different parts of the trees. From 55 trees, the majority of the pathogens were isolated from the scion shoot (36 trees), followed by the pruning wound on the rootstock ( 5 trees), the rootstock (10 trees), and, finally, from the bud union ( 6 trees). Internal and external symptoms associated with canker on the scion shoot of the tree are illustrated in Figure 1. One pathogen (Fig. 1B, C, D) and in some cases more than one pathogen (Fig. 1E, F) was isolated and, more importantly on some trees, canker and wood rot symptoms occurred on more than one plant part (Fig. 1E, F). Multiple infections were often on the scion shoot and from the pruning wound on the rootstock. Fruiting bodies (pycnidia) of Ascomycete fungi, specifically Coniochaeta spp., D. rubi-ulmifolii 
Table 1. List of isolates identified in this study

\begin{tabular}{|c|c|c|c|c|c|c|c|c|}
\hline \multirow[b]{2}{*}{ Group, strain ${ }^{u}$} & \multirow[b]{2}{*}{ Species } & \multirow[b]{2}{*}{$\operatorname{Area}^{\mathbf{v}}$} & \multirow[b]{2}{*}{ Cultivar } & \multirow[b]{2}{*}{ Plant part } & \multicolumn{3}{|c|}{ GenBank accession ${ }^{t}$} & \multirow{2}{*}{$\begin{array}{l}\text { Support } \\
(\%)^{\mathbf{w}}\end{array}$} \\
\hline & & & & & ITS & $\mathbf{E F}$ & $\beta$-Tubulin & \\
\hline \multicolumn{9}{|c|}{ Amphisphaeriaceae } \\
\hline STEU 8283 & $\begin{array}{r}\text { Truncatella } \\
\text { angustata }\end{array}$ & G & African Red & $\begin{array}{l}\text { Canker on scion shoot } \\
\text { of 1-year-old tree }\end{array}$ & KY312617 & $\cdots$ & $\cdots$ & 98 \\
\hline STEU 8284 & T. angustata & $\mathrm{K}$ & Golden Delicious & $\begin{array}{l}\text { Bud union of a } \\
\text { nursery tree }\end{array}$ & KY312618 & $\ldots$ & $\cdots$ & $\cdots$ \\
\hline \multicolumn{9}{|l|}{ Basidiomycetes } \\
\hline STEU 8285 & Bjerkandera sp. & $\mathrm{R}$ & Royal Beaut/MM109 & $\begin{array}{l}\text { Pruning wound on } \\
\text { rootstock of a } \\
\text { nursery tree }\end{array}$ & KY312619 & $\cdots$ & $\cdots$ & $87^{x}$ \\
\hline STEU 8286 & Bjerkandera sp. & $\mathrm{R}$ & Royal Beaut/M793 & $\begin{array}{l}\text { Soft rot in pruning } \\
\text { wound of nursery } \\
\text { tree }\end{array}$ & KY312620 & $\cdots$ & $\cdots$ & $\cdots$ \\
\hline STEU 8287 & $\begin{array}{l}\text { Chondrostereum } \\
\text { purpureum }\end{array}$ & $\mathrm{K}$ & Rosy Glow & $\begin{array}{l}\text { Pruning wound on a } \\
\text { mature tree }\end{array}$ & KY312621 & $\cdots$ & $\cdots$ & 100 \\
\hline STEU 8288 & Peniophora sp. 2 & $\mathrm{~K}$ & Golden Delicious & $\begin{array}{l}\text { Canker on scion shoot } \\
\text { of 1-year-old tree }\end{array}$ & KY312622 & $\cdots$ & $\cdots$ & 96 \\
\hline STEU 8289 & Peniophora sp. 2 & $\mathrm{~L}$ & Golden Delicious/M793 & $\begin{array}{l}\text { Bud union of a } \\
\text { nursery tree }\end{array}$ & KY312623 & $\ldots$ & $\cdots$ & $\cdots$ \\
\hline STEU 8290 & Peniophora sp. 1 & $\mathrm{~K}$ & Rosy Glow & $\begin{array}{l}\text { Pruning wound on a } \\
\text { mature tree }\end{array}$ & KY312624 & $\cdots$ & $\cdots$ & 100 \\
\hline STEU 8291 & Peniophora sp. 1 & $\mathrm{~L}$ & Golden Delicious/M793 & $\begin{array}{l}\text { Bud union of a } \\
\text { nursery tree }\end{array}$ & KY312625 & $\cdots$ & $\cdots$ & $\cdots$ \\
\hline STEU 8292 & $\begin{array}{l}\text { Schizophyllum } \\
\text { commune }\end{array}$ & $\mathrm{L}$ & Golden Delicious/M793 & $\begin{array}{l}\text { Pruning wound on } \\
\text { rootstock of } \\
\text { nursery tree }\end{array}$ & KY312626 & $\cdots$ & $\cdots$ & 99 \\
\hline STEU 8293 & S. commune & $\mathrm{K}$ & Royal Beaut & $\begin{array}{l}\text { Canker on scion shoot } \\
\text { of 1-year-old tree }\end{array}$ & KY312627 & $\cdots$ & $\cdots$ & $\cdots$ \\
\hline STEU 8294 & Stereum hirsutum & $\mathrm{K}$ & Royal Beaut & $\begin{array}{l}\text { Canker on scion shoot } \\
\text { of 1-year-old tree }\end{array}$ & KY312628 & $\cdots$ & $\cdots$ & 74 \\
\hline STEU 8295 & $\begin{array}{l}\text { Trametes } \\
\text { versicolor }\end{array}$ & $\mathrm{L}$ & Golden Delicious/MM109 & $\begin{array}{l}\text { Wound on rootstock } \\
\text { of nursery tree }\end{array}$ & KY312629 & $\cdots$ & $\cdots$ & 79 \\
\hline STEU 8296 & T. versicolor & $\mathrm{L}$ & Golden Delicious/MM110 & $\begin{array}{l}\text { Bud union of a } \\
\text { nursery tree }\end{array}$ & KY312630 & $\cdots$ & $\cdots$ & $\cdots$ \\
\hline \multicolumn{9}{|c|}{ Botryosphaeriaceae } \\
\hline STEU 8297 & $\begin{array}{l}\text { Botryosphaeria } \\
\text { dothidea }\end{array}$ & $\mathrm{R}$ & Gale Gala/G222 & $\begin{array}{l}\text { Bud union of a } \\
\text { nursery tree }\end{array}$ & $\cdots$ & KY312606 & $\cdots$ & 73 \\
\hline STEU 8298 & B. dothidea & $\mathrm{R}$ & Royal Beaut/M793 & $\begin{array}{l}\text { Bud union of a } \\
\text { nursery tree }\end{array}$ & $\cdots$ & KY312607 & $\cdots$ & $\cdots$ \\
\hline STEU 8299 & Diplodia seriata & $\mathrm{K}$ & Early Red One & Bud on new shoot & $\ldots$ & KY312608 & $\ldots$ & 79 \\
\hline STEU 8300 & D. seriata & $\mathrm{R}$ & Royal Beaut/M793 & $\begin{array}{l}\text { Bud union of a } \\
\text { nursery tree }\end{array}$ & $\cdots$ & KY312609 & $\ldots$ & $\cdots$ \\
\hline STEU 8301 & $\begin{array}{l}\text { Neofusicoccum } \\
\text { austral }\end{array}$ & $\mathrm{R}$ & Royal Beaut/MM109 & $\begin{array}{l}\text { Bud union of a } \\
\text { nursery tree }\end{array}$ & $\cdots$ & KY312610 & $\ldots$ & 80 \\
\hline STEU 8302 & N. austral & $\mathrm{R}$ & Gale Gala/G222 & $\begin{array}{l}\text { Wound on scion shoot } \\
\text { of nursery tree }\end{array}$ & $\cdots$ & KY312611 & $\cdots$ & $\cdots$ \\
\hline STEU 8303 & $\begin{array}{l}\text { Neofusicoccum } \\
\text { sp. } 4\end{array}$ & $\mathrm{R}$ & Gale Gala/G222 & $\begin{array}{l}\text { Pruning wound on } \\
\text { rootstock of a } \\
\text { nursery tree }\end{array}$ & $\cdots$ & KY312612 & $\ldots$ & 59 \\
\hline STEU 8304 & N. viticlavatum & $\mathrm{K}$ & Fuji Supreme & $\begin{array}{c}\text { Canker on rootstock } \\
\text { of } 1 \text {-year-old tree }\end{array}$ & $\cdots$ & KY312613 & $\ldots$ & 91 \\
\hline STEU 8305 & N. viticlavatum & $\mathrm{K}$ & Fuji Supreme & $\begin{array}{l}\text { Canker on rootstock of } \\
1 \text {-year-old tree }\end{array}$ & $\cdots$ & KY312614 & $\cdots$ & $\cdots$ \\
\hline STEU 8306 & $\begin{array}{l}N . \\
\quad \text { stellenboschiana }\end{array}$ & $\mathrm{R}$ & Gale Gala/G222 & $\begin{array}{l}\text { Pruning wound } \\
\text { on rootstock } \\
\text { of a nursery } \\
\text { tree }\end{array}$ & $\cdots$ & KY312615 & $\cdots$ & 62 \\
\hline STEU 8307 & $\begin{array}{l}N . \\
\text { stellenboschiana }\end{array}$ & $\mathrm{R}$ & Gale Gala/G223 & $\begin{array}{l}\text { Pruning wound on } \\
\text { rootstock of a } \\
\text { nursery tree }\end{array}$ & $\ldots$ & KY312616 & $\cdots$ & $\cdots$ \\
\hline
\end{tabular}

t ITS = internal transcribed spacer and EF = elongation factor.

$\mathrm{u}$ Taxonomic group and strain number.

v Area sampled: $\mathrm{G}=$ Grabouw, $\mathrm{H}=$ Hermanus, $\mathrm{K}=$ Kouebokkeveld, $\mathrm{L}=$ Langkloof, and $\mathrm{R}=$ Riviersonderend.

${ }^{w}$ Bootstrap support value.

x Isolate clades with Bjerkandera adusta, B. fumosa, and B. atroalaba. Isolates cannot be identified up to species level.

y The isolates formed a clade with Cytospora malicola (DQ243802) found on an apple tree in Michigan and C. germanica found from Populus sp. in South Africa (DQ243793).

${ }^{\mathrm{z}}$ D. eres is a species complex that has been designated into two groups (A and B) (Guarnaccia et al. 2018). 
Table 1. (Continued from previous page)

\begin{tabular}{|c|c|c|c|c|c|c|c|c|}
\hline \multirow[b]{2}{*}{ Group, strain ${ }^{u}$} & \multirow[b]{2}{*}{ Species } & \multirow[b]{2}{*}{ Area $^{v}$} & \multirow[b]{2}{*}{ Cultivar } & \multirow[b]{2}{*}{ Plant part } & \multicolumn{3}{|c|}{ GenBank accession ${ }^{t}$} & \multirow{2}{*}{$\underset{(\%)^{\mathrm{w}}}{\text { Support }}$} \\
\hline & & & & & ITS & EF & $\beta$-Tubulin & \\
\hline \multicolumn{9}{|l|}{ Cadophora } \\
\hline STEU 8308 & $\begin{array}{l}\text { Cadophora luteo- } \\
\text { olivacea }\end{array}$ & $\mathrm{K}$ & Royal Beaut/MM109 & $\begin{array}{l}\text { Pruning wound on } \\
\text { rootstock of a nursery } \\
\text { tree }\end{array}$ & KY312631 & $\cdots$ & $\cdots$ & 88 \\
\hline STEU 8309 & C. luteo-olivacea & $\mathrm{K}$ & Golden Delicious/M793 & $\begin{array}{l}\text { Pruning wound on } \\
\text { rootstock of a nursery } \\
\text { tree }\end{array}$ & KY312632 & $\ldots$ & $\cdots$ & $\cdots$ \\
\hline STEU 8310 & Cadophora sp. & $\mathrm{K}$ & Golden Delicious/M793 & $\begin{array}{l}\text { Bud union of a nursery } \\
\text { tree }\end{array}$ & KY312633 & $\ldots$ & $\ldots$ & 96 \\
\hline STEU 8311 & Cadophora sp. & $\mathrm{K}$ & Golden Delicious/M793 & Bud union of a nursery tree & KY312634 & $\ldots$ & $\ldots$ & $\ldots$ \\
\hline \multicolumn{9}{|l|}{ Coniochaeta } \\
\hline STEU 8312 & $\begin{array}{r}\text { Coniochaeta } \\
\text { fasciculata }\end{array}$ & $\mathrm{K}$ & Gale Gala/CG4202 & $\begin{array}{l}\text { Pruning wound on } \\
\text { rootstock of a nursery } \\
\text { tree }\end{array}$ & KY312635 & $\ldots$ & $\cdots$ & 100 \\
\hline STEU 8313 & C. fasciculata & $\mathrm{K}$ & Golden Delicious & $\begin{array}{l}\text { Canker on scion shoot } \\
\text { of } 1 \text {-year-old tree }\end{array}$ & KY312636 & $\ldots$ & $\cdots$ & $\cdots$ \\
\hline STEU 8314 & C. velutina & $\mathrm{R}$ & Gale Gala/G222 & $\begin{array}{l}\text { Pruning wound on } \\
\text { rootstock of a nursery } \\
\text { tree }\end{array}$ & KY312637 & $\cdots$ & $\cdots$ & 70 \\
\hline STEU 8315 & C. velutina & $\mathrm{K}$ & Early Red One & $\begin{array}{l}\text { Pruning wound on a } \\
\text { mature tree }\end{array}$ & KY312638 & $\ldots$ & $\cdots$ & $\cdots$ \\
\hline STEU 8316 & Coniochaeta sp. & G & Golden Delicious & $\begin{array}{l}\text { Fruiting body on canker } \\
\text { found on a 1-year-old } \\
\text { tree }\end{array}$ & KY312639 & $\cdots$ & $\cdots$ & $\cdots$ \\
\hline \multicolumn{9}{|l|}{ Cytospora } \\
\hline STEU 8317 & $\begin{array}{l}\text { Cytospora } \\
\quad \text { malicolal } \\
\text { germanica } \\
\text { complex }\end{array}$ & $\mathrm{K}$ & M793 & $\begin{array}{l}\text { Canker on rooted } \\
\text { rootstock cutting }\end{array}$ & KY312640 & $\cdots$ & $\cdots$ & $58^{\mathrm{y}}$ \\
\hline STEU 8318 & $\begin{array}{l}\text { C. malicolal } \\
\text { germanica } \\
\text { complex }\end{array}$ & K & MM109 & $\begin{array}{l}\text { Canker on rooted } \\
\text { rootstock cutting }\end{array}$ & KY312641 & $\ldots$ & $\ldots$ & $\ldots$ \\
\hline \multicolumn{9}{|l|}{ Diaporthe } \\
\hline STEU 8319 & $\begin{array}{l}\text { Diaporthe } \\
\text { ambigua }\end{array}$ & $\mathrm{K}$ & Early Red One & $\begin{array}{l}\text { Pruning wound on a } \\
\text { mature tree }\end{array}$ & KY312642 & MH925324 & $\cdots$ & 61 \\
\hline STEU 8320 & D. ambigua & K & Rosy Glow & $\begin{array}{l}\text { Pruning wound on a } \\
\text { mature tree }\end{array}$ & KY312643 & MH925323 & $\cdots$ & $\cdots$ \\
\hline STEU 8321 & D. cynaroidis & $\mathrm{K}$ & Gale Gala/G222 & $\begin{array}{l}\text { Wound on rootstock } \\
\text { of a nursery tree }\end{array}$ & KY312644 & MH925322 & $\ldots$ & 99 \\
\hline STEU 8322 & D. eres (B) & $\mathrm{R}$ & Gale Gala/G222 & $\begin{array}{l}\text { Pruning wound on } \\
\text { rootstock of a nursery } \\
\text { tree }\end{array}$ & KY312645 & MH925321 & $\ldots$ & $62^{z}$ \\
\hline STEU 8323 & D. eres (B) & G & Sundowner & $\begin{array}{l}\text { Canker on scion shoot } \\
\text { of a 1-year-old tree }\end{array}$ & KY312646 & MH925320 & $\ldots$ & $\ldots$ \\
\hline STEU 8324 & D. foeniculina & $\mathrm{G}$ & Golden Delicious & $\begin{array}{l}\text { Pruning wound on a } \\
\text { mature tree }\end{array}$ & KY312647 & MH925319 & $\ldots$ & 88 \\
\hline STEU 8325 & D. foeniculina & $\mathrm{R}$ & Royal Beaut/M793 & $\begin{array}{l}\text { Pruning wound on } \\
\text { rootstock of a nursery } \\
\text { tree }\end{array}$ & KY312648 & MH925318 & $\cdots$ & $\cdots$ \\
\hline STEU 8326 & D. eres (A) & $\mathrm{K}$ & Golden Delicious/MM109 & $\begin{array}{l}\text { Bud union of a nursery } \\
\text { tree }\end{array}$ & KY312649 & $\ldots$ & $\ldots$ & 59 \\
\hline STEU 8327 & D. $\operatorname{eres}(\mathrm{A})$ & $\mathrm{H}$ & Cripps Red & $\begin{array}{l}\text { Canker on scion shoot of } \\
\text { a 1-year-old tree }\end{array}$ & KY312650 & MH925317 & $\ldots$ & $\ldots$ \\
\hline STEU 8328 & D. virgiliae & $\mathrm{K}$ & Golden Delicious/MM109 & $\begin{array}{l}\text { Bud union of a nursery } \\
\text { tree }\end{array}$ & KY312651 & MH925316 & $\ldots$ & 100 \\
\hline STEU 8329 & D. virgiliae & $\mathrm{L}$ & Golden Delicious/M793 & $\begin{array}{l}\text { Wound on rootstock } \\
\text { of a nursery tree }\end{array}$ & KY312652 & MH925315 & $\ldots$ & $\ldots$ \\
\hline STEU 8330 & D. amygdali & $\mathrm{K}$ & Royal Beaut/MM109 & $\begin{array}{l}\text { Bud union of a nursery } \\
\text { tree }\end{array}$ & KY312653 & MH925314 & $\ldots$ & 66 \\
\hline STEU 8331 & D. amygdali & $\mathrm{K}$ & Royal Beaut/MM110 & $\begin{array}{l}\text { Wound on rootstock } \\
\text { of a nursery tree }\end{array}$ & KY312654 & MH925313 & $\cdots$ & $\cdots$ \\
\hline \multicolumn{9}{|l|}{ Diatrypaceae } \\
\hline STEU 8333 & Eutypa lata & $\mathrm{K}$ & Golden Delicious & $\begin{array}{l}\text { Pruning wound on a } \\
\text { mature tree }\end{array}$ & $\ldots$ & $\ldots$ & $\begin{array}{l}\text { MH925330 } \\
\text { (Continued on }\end{array}$ & $\begin{array}{c}100 \\
\text { next page) }\end{array}$ \\
\hline
\end{tabular}


Table 1. (Continued from previous page)

\begin{tabular}{|c|c|c|c|c|c|c|c|c|}
\hline \multirow[b]{2}{*}{ Group, strainu } & \multirow[b]{2}{*}{ Species } & \multirow[b]{2}{*}{ Areav $^{\mathbf{v}}$} & \multirow[b]{2}{*}{ Cultivar } & \multirow[b]{2}{*}{ Plant part } & \multicolumn{3}{|c|}{ GenBank accession $^{t}$} & \multirow{2}{*}{$\begin{array}{l}\text { Support } \\
(\%)^{\mathbf{w}}\end{array}$} \\
\hline & & & & & ITS & EF & $\beta$-Tubulin & \\
\hline STEU 8334 & E. lata & $\mathrm{K}$ & Golden Delicious/M793 & $\begin{array}{l}\text { Bud union of a nursery } \\
\text { tree }\end{array}$ & $\cdots$ & $\cdots$ & MH925329 & $\cdots$ \\
\hline STEU 8335 & E. cremea & $\mathrm{G}$ & Golden Delicious & $\begin{array}{l}\text { Pruning wound on a } \\
\text { mature tree }\end{array}$ & $\cdots$ & $\cdots$ & MH925328 & 70 \\
\hline STEU 8336 & E. cremea & $\mathrm{G}$ & Golden Delicious & $\begin{array}{l}\text { Pruning wound on a } \\
\text { mature tree }\end{array}$ & $\cdots$ & $\cdots$ & MH925327 & $\cdots$ \\
\hline STEU 8337 & Eutypella citricola & $\mathrm{R}$ & Royal Beaut/MM109 & $\begin{array}{l}\text { Wound on scion shoot } \\
\text { of a nursery tree }\end{array}$ & $\cdots$ & $\cdots$ & MH925326 & 100 \\
\hline STEU 8338 & E. citricola & $\mathrm{G}$ & Golden Delicious & $\begin{array}{c}\text { Canker on scion shoot } \\
\text { of a 1-year-old tree }\end{array}$ & $\cdots$ & $\cdots$ & MH925325 & $\cdots$ \\
\hline \multicolumn{9}{|l|}{ Dothideomycetes } \\
\hline STEU 8339 & $\begin{array}{l}\text { Didymella } \\
\text { pomorum }\end{array}$ & $\mathrm{K}$ & Golden Delicious & $\begin{array}{l}\text { Canker on scion shoot } \\
\text { of a 1-year-old tree }\end{array}$ & KY312661 & $\cdots$ & $\ldots$ & 66 \\
\hline STEU 8340 & D. pomorum & $\mathrm{K}$ & M793 & Rooted rootstock cutting & KY312662 & $\ldots$ & $\ldots$ & $\ldots$ \\
\hline STEU 8341 & D. pomorum & $\mathrm{K}$ & Golden Delicious/M793 & $\begin{array}{l}\text { Bud union of a nursery } \\
\text { tree }\end{array}$ & KY312663 & $\cdots$ & $\cdots$ & $\cdots$ \\
\hline STEU 8342 & D. pomorum & $\mathrm{K}$ & M793 & Rooted rootstock cutting & KY312664 & $\ldots$ & $\ldots$ & $\ldots$ \\
\hline STEU 8343 & $\begin{array}{l}\text { Didymosphaeria } \\
\text { variabile }\end{array}$ & $\mathrm{H}$ & Cripps Red & $\begin{array}{l}\text { Canker on scion shoot } \\
\text { of a 1-year-old tree }\end{array}$ & KY312665 & $\cdots$ & $\cdots$ & 94 \\
\hline STEU 8344 & D. variabile & $\mathrm{G}$ & Golden Delicious & $\begin{array}{l}\text { Pruning wound on a } \\
\text { mature tree }\end{array}$ & KY312666 & $\cdots$ & $\cdots$ & $\cdots$ \\
\hline STEU 8345 & $\begin{array}{l}\text { D. rubi-ulmifolii } \\
\text { sensu lato }\end{array}$ & $\mathrm{R}$ & Gale Gala/G222 & $\begin{array}{l}\text { Bud union of a } \\
\text { nursery tree }\end{array}$ & KY312667 & $\cdots$ & $\cdots$ & 80 \\
\hline STEU 8346 & $\begin{array}{l}\text { D. rubi-ulmifolii } \\
\text { sensu lato }\end{array}$ & $\mathrm{K}$ & Golden Delicious/M793 & $\begin{array}{l}\text { Wound on rootstock } \\
\text { of a nursery tree }\end{array}$ & KY312668 & $\cdots$ & $\cdots$ & $\cdots$ \\
\hline \multicolumn{9}{|c|}{ Phaeoacremonium } \\
\hline STEU 8347 & $\begin{array}{l}\text { Phaeoacremonium } \\
\text { australiense }\end{array}$ & $\mathrm{K}$ & Early Red One & $\begin{array}{l}\text { Pruning wound on a } \\
\text { mature tree }\end{array}$ & $\cdots$ & $\cdots$ & KY312669 & 93 \\
\hline STEU 8348 & P. australiense & $\mathrm{G}$ & Golden Delicious & $\begin{array}{l}\text { Pruning wound on a } \\
\text { mature tree }\end{array}$ & $\cdots$ & $\cdots$ & KY312670 & $\cdots$ \\
\hline STEU 8349 & P. austroafricanum & $\mathrm{L}$ & Golden Delicious/M793 & $\begin{array}{l}\text { Bud union of a nursery } \\
\text { tree }\end{array}$ & $\cdots$ & $\cdots$ & KY312671 & 100 \\
\hline STEU 8350 & P. austroafricanum & $\mathrm{L}$ & Golden Delicious/M793 & $\begin{array}{l}\text { Bud union of a nursery } \\
\text { tree }\end{array}$ & $\cdots$ & $\cdots$ & KY312672 & $\cdots$ \\
\hline STEU 8351 & $\begin{array}{l}\text { P. fraxinopenn- } \\
\text { sylvanicum }\end{array}$ & $\mathrm{K}$ & Golden Delicious & $\begin{array}{l}\text { Pruning wound on a } \\
\text { mature tree }\end{array}$ & $\cdots$ & $\cdots$ & KY312673 & 99 \\
\hline STEU 8352 & $\begin{array}{l}\text { P. fraxinopenn- } \\
\text { sylvanicum }\end{array}$ & $\mathrm{L}$ & Golden Delicious/MM109 & $\begin{array}{l}\text { Bud union of a nursery } \\
\text { tree }\end{array}$ & $\cdots$ & $\cdots$ & KY312674 & $\cdots$ \\
\hline STEU 8353 & P. inflatipes & $\mathrm{K}$ & Early Red One & $\begin{array}{l}\text { Pruning wound on a } \\
\text { mature tree }\end{array}$ & $\cdots$ & $\cdots$ & KY312675 & 100 \\
\hline STEU 8354 & P. inflatipes & $\mathrm{K}$ & Early Red One & $\begin{array}{l}\text { Pruning wound on a } \\
\text { mature tree }\end{array}$ & $\cdots$ & $\cdots$ & KY312676 & $\cdots$ \\
\hline STEU 8355 & P. iranianum & $\mathrm{G}$ & Golden Delicious/M793 & $\begin{array}{l}\text { Pruning wound on a } \\
\text { nursery tree }\end{array}$ & $\cdots$ & $\cdots$ & KY312677 & 100 \\
\hline STEU 8356 & P. minimum & $\mathrm{G}$ & Golden Delicious & $\begin{array}{l}\text { Pruning wound on a } \\
\text { mature tree }\end{array}$ & $\cdots$ & $\cdots$ & KY312678 & 100 \\
\hline STEU 8357 & P. minimum & G & Golden Delicious & $\begin{array}{l}\text { Pruning wound on a } \\
\text { mature tree }\end{array}$ & $\cdots$ & $\cdots$ & KY312679 & $\cdots$ \\
\hline STEU 8358 & P. prunicola & $\mathrm{K}$ & Early Red One & $\begin{array}{l}\text { Pruning wound on a } \\
\text { mature tree }\end{array}$ & $\cdots$ & $\cdots$ & KY312680 & 100 \\
\hline STEU 8359 & P. prunicola & $\mathrm{G}$ & Golden Delicious & $\begin{array}{l}\text { Pruning wound on a } \\
\text { mature tree }\end{array}$ & $\cdots$ & $\cdots$ & KY312681 & $\cdots$ \\
\hline STEU 8360 & P. scolyti & $\mathrm{K}$ & Early Red One & $\begin{array}{l}\text { Pruning wound on a } \\
\text { mature tree }\end{array}$ & $\ldots$ & $\cdots$ & KY312682 & 99 \\
\hline STEU 8361 & P. subulatum & $\mathrm{K}$ & Gale Gala/CG4202 & $\begin{array}{l}\text { Canker on scion shoot } \\
\text { of a 1-year-old tree }\end{array}$ & $\cdots$ & $\cdots$ & KY312683 & 100 \\
\hline STEU 8362 & P. subulatum & $\mathrm{K}$ & Gale Gala/CG4202 & $\begin{array}{l}\text { Pruning wound on a } \\
\text { nursery tree }\end{array}$ & $\cdots$ & $\cdots$ & KY312684 & $\cdots$ \\
\hline STEU 8363 & P. viticola & $\mathrm{K}$ & Early Red One & $\begin{array}{l}\text { Pruning wound on a } \\
\text { mature tree }\end{array}$ & $\cdots$ & $\cdots$ & KY312685 & 80 \\
\hline STEU 8364 & P. viticola & K & Early Red One & $\begin{array}{l}\text { Pruning wound on a } \\
\text { mature tree }\end{array}$ & $\cdots$ & $\cdots$ & KY312686 & $\cdots$ \\
\hline STEU 8402 & P. geminum & $\mathrm{K}$ & Royal Beaut/M793 & $\begin{array}{l}\text { Pruning wound on a } \\
\text { nursery tree }\end{array}$ & $\cdots$ & $\cdots$ & KY312689 & 100 \\
\hline STEU 8401 & P. longicollarum & $\mathrm{L}$ & Golden Delicious/M793 & $\begin{array}{l}\text { Bud union of a nursery } \\
\text { tree }\end{array}$ & $\cdots$ & $\cdots$ & KY312688 & 100 \\
\hline STEU 8400 & P. longicollarum & $\mathrm{K}$ & Rosy Glow & $\begin{array}{l}\text { Pruning wound on a } \\
\text { mature tree }\end{array}$ & $\cdots$ & $\cdots$ & KY312687 & $\cdots$ \\
\hline
\end{tabular}


Table 2. Incidence (number of infected plant parts) of fungal species isolated from apple propagation material, nursery trees, and 1-year-old orchards

\begin{tabular}{|c|c|c|c|c|c|c|}
\hline \multirow[b]{3}{*}{ Taxonomic group, species } & \multirow[b]{3}{*}{ Infected $^{\mathbf{v}}$} & \multicolumn{5}{|c|}{ Incidence for specific material source } \\
\hline & & \multirow[b]{2}{*}{ Orchards $^{w}$} & \multirow[b]{2}{*}{ Trees $^{x}$} & \multirow[b]{2}{*}{ Scion blocks ${ }^{y}$} & \multicolumn{2}{|c|}{ Mother blocks ${ }^{\mathbf{u}}$} \\
\hline & & & & & Layer & Nursery \\
\hline \multicolumn{7}{|l|}{ Amphisphaeriaceae } \\
\hline Truncatella angustata ${ }^{\mathrm{z}}$ & 16 & 1 & 5 & 0 & 10 & 0 \\
\hline \multicolumn{7}{|l|}{ Basidiomycetes } \\
\hline Bjerkandera sp. & 8 & 0 & 7 & 1 & 0 & 0 \\
\hline Chondrostereum purpureum ${ }^{\mathrm{z}}$ & 1 & 0 & 0 & 1 & 0 & 0 \\
\hline Peniophora sp. 2 & 4 & 1 & 3 & 0 & 0 & 0 \\
\hline Peniophora sp. 1 & 6 & 1 & 3 & 2 & 0 & 0 \\
\hline Schizophyllum commune $e^{\mathrm{z}}$ & 16 & 4 & 10 & 1 & 0 & 1 \\
\hline Stereum hirsutum $^{\mathrm{z}}$ & 1 & 1 & 0 & 0 & 0 & 0 \\
\hline Trametes versicolor $^{\mathrm{Z}}$ & 37 & 2 & 18 & 17 & 0 & 0 \\
\hline \multicolumn{7}{|l|}{ Botryosphaeriaceae } \\
\hline Botryosphaeria dothidea ${ }^{\mathrm{Z}}$ & 9 & 0 & 7 & 0 & 1 & 1 \\
\hline Diplodia seriata ${ }^{\mathrm{z}}$ & 78 & 11 & 32 & 17 & 0 & 18 \\
\hline Neofusicoccum australe $^{\mathrm{z}}$ & 8 & 2 & 6 & 0 & 0 & 0 \\
\hline Neofusicoccum sp. $4^{\mathrm{z}}$ & 1 & 0 & 1 & 0 & 0 & 0 \\
\hline N. stellenboschiana & 2 & 0 & 2 & 0 & 0 & 0 \\
\hline N. viticlavatum & 1 & 1 & 0 & 0 & 0 & 0 \\
\hline \multicolumn{7}{|l|}{ Cadophora spp. } \\
\hline Cadophora luteo-olivacea ${ }^{\mathrm{z}}$ & 17 & 0 & 17 & 0 & 0 & 0 \\
\hline Cadophora sp. & 6 & 0 & 6 & 0 & 0 & 0 \\
\hline \multicolumn{7}{|l|}{ Coniochaeta spp. } \\
\hline Coniochaeta fasciculata & 18 & 3 & 15 & 0 & 0 & 0 \\
\hline C. velutina & 13 & 1 & 10 & 2 & 0 & 0 \\
\hline Coniochaeta sp. & 1 & 1 & 0 & 0 & 0 & 0 \\
\hline \multicolumn{7}{|l|}{ Cytospora spp. } \\
\hline Cytospora malicola/germanica complex ${ }^{\mathrm{Z}}$ & 12 & 0 & 0 & 0 & 0 & 12 \\
\hline \multicolumn{7}{|l|}{ Diaporthe spp. } \\
\hline Diaporthe ambigua ${ }^{\mathrm{z}}$ & 3 & 0 & 0 & 2 & 1 & 0 \\
\hline D. cynaroidis & 3 & 0 & 3 & 0 & 0 & 0 \\
\hline D. eres $(\mathrm{B})^{\mathrm{z}}$ & 33 & 2 & 30 & 1 & 0 & 0 \\
\hline D. foeniculina ${ }^{\mathrm{z}}$ & 7 & 2 & 1 & 3 & 1 & 0 \\
\hline D. $\operatorname{eres}(\mathrm{A})^{\mathrm{z}}$ & 2 & 1 & 1 & 0 & 0 & 0 \\
\hline D. virgiliae & 10 & 0 & 10 & 0 & 0 & 0 \\
\hline D. amygdali & 2 & 0 & 2 & 0 & 0 & 0 \\
\hline \multicolumn{7}{|l|}{ Diatrypaceae } \\
\hline Eutypa lata & 82 & 0 & 5 & 77 & 0 & 0 \\
\hline E. cremea & 8 & 0 & 0 & 8 & 0 & 0 \\
\hline Eutypella citricola & 9 & 5 & 1 & 3 & 0 & 0 \\
\hline \multicolumn{7}{|l|}{ Didymella spp. } \\
\hline Didymella pomorum ${ }^{\mathrm{z}}$ & 94 & 3 & 34 & 1 & 55 & 1 \\
\hline Didymosphaeria spp. & & & & & & \\
\hline Didymosphaeria variabile ${ }^{\mathrm{z}}$ & 29 & 2 & 18 & 8 & 1 & 0 \\
\hline D. rubi-ulmifolii sensu lato & 276 & 15 & 241 & 13 & 7 & 0 \\
\hline Phaeoacremonium spp. & & & & & & \\
\hline Phaeoacremonium australiense & 1 & 0 & 0 & 1 & 0 & 0 \\
\hline P. austroafricanum & 18 & 1 & 15 & 2 & 0 & 0 \\
\hline P. fraxinopennsylvanicum ${ }^{\mathrm{z}}$ & 19 & 1 & 3 & 15 & 0 & 0 \\
\hline P. inflatipes & 1 & 0 & 0 & 1 & 0 & 0 \\
\hline P. iranianum ${ }^{\mathrm{z}}$ & 7 & 0 & 7 & 0 & 0 & 0 \\
\hline P. minimum ${ }^{\mathrm{z}}$ & 13 & 0 & 2 & 11 & 0 & 0 \\
\hline P. prunicola & 3 & 0 & 0 & 3 & 0 & 0 \\
\hline P. scolyti & 1 & 0 & 0 & 1 & 0 & 0 \\
\hline P. subulatum & 5 & 0 & 5 & 0 & 0 & 0 \\
\hline P. viticola ${ }^{\mathrm{z}}$ & 16 & 0 & 0 & 15 & 0 & 1 \\
\hline P. geminum & 2 & 0 & 2 & 0 & 0 & 0 \\
\hline P. longicollarum & 1 & 0 & 0 & 1 & 0 & 0 \\
\hline
\end{tabular}

u Rootstock mother blocks. Plant parts included rootstock shoots only for 405 asymptomatic layer block shoots and 87 symptomatic 1-year-old rootstock shoots.

v Total number of infected plant parts, including 1-year-old orchards, nursery trees, scion mother blocks, and rootstock mother blocks.

${ }^{w}$ Plant parts from 130 trees included cankers forming on scion shoots, pruning wounds on rootstocks, bud unions, or rootstocks of 1-year-old diseased orchard trees.

x Plant parts from 480 trees included scion shoots, pruning wounds on rootstocks, bud unions, or rootstocks.

y Plant parts from 310 trees included cankers, pruning wounds, or scion shoots.

z Previously reported on apple trees (Farr and Rossman 2019). 
sensu lato, and Didymella pomorum, were found on the cankers that formed on the scion shoot. The fruiting structures of $S$. commune were found on one of the dead Royal Beaut trees.

Certified nursery apple trees. In total 480 certified healthy nursery trees were collected of which $312(65 \%)$ harbored possible canker or wood rot pathogens. The nursery trees did not exhibit any external symptoms; however, a longitudinal section through the bud union and pruning wound often show vascular discoloration and/or soft rot (Fig. 1G, H, I). Thirty-two different fungal taxa were isolated from these nursery trees (Table 2). D. rubi-ulmifolii sensu lato was isolated from
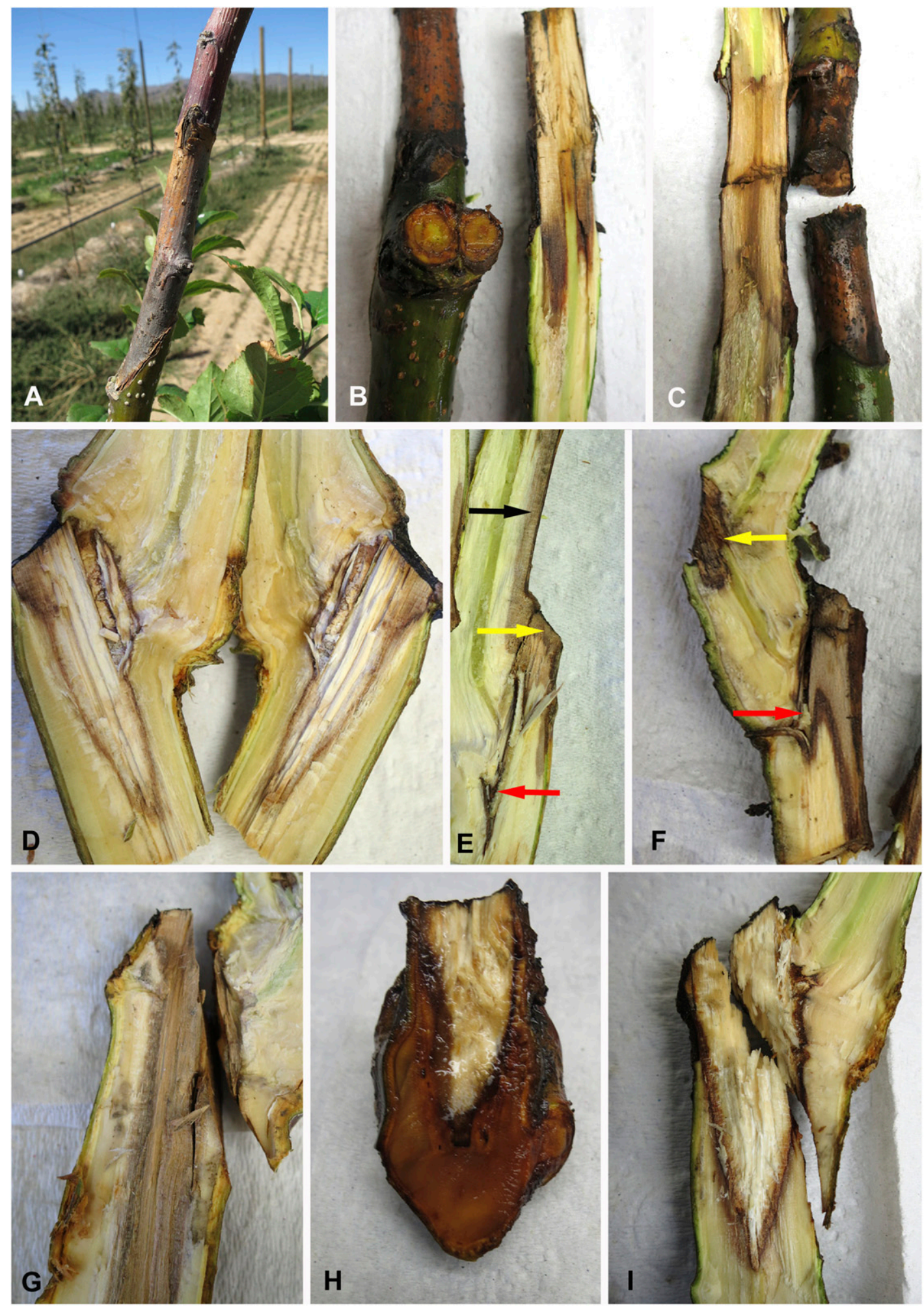

Fig. 1. External and internal symptoms exhibited on 1-year-old apple trees (A-F). A, Typical canker forming on the scion shoot of a 1-year-old apple tree. B and C, Sections through the scion shoots and D, a pruning wound show vascular discoloration caused by B, Diplodia seriata; C, Didymosphaeria rubi-ulmifolii sensu lato; and D, Trametes versicolor. More than one pathogen was isolated from sections through cankers and pruning wounds. E, Causal organisms involved in the symptom expression were Phaeoacremonium fraxinopennsylvanicum isolated from the bud union (red arrow) whereas Coniochaeta fasciculata (yellow arrow) and Diplodia seriata (black arrow) were isolated from the canker that formed on the scion shoot. F, P. austroafricanum was isolated from the bud union (red arrow) and Diplodia seriata isolated from the pruning wound (yellow arrow). Cross sections through the pruning wound and the bud union of external asymptomatic nursery apple trees (G-I). Sections through the pruning wound and bud union showing G, brown internal discoloration caused by Didymosphaeria rubi-ulmifolii sensu lato and white rot symptoms caused by H, Bjerkandera adusta and I, Trametes versicolor. 
274 plant parts and was the predominant fungus isolated from the nursery material. D. rubi-ulmifolii sensu lato was also the dominant pathogen isolated from all four plant parts. D. seriata, Diaporthe eres (B), D. pomorum, T. versicolor, D. variabile, Cadophora luteoolivacea, P. austroafricanum, Coniochaeta velutina, S. commune, $C$. fasciculata, and $D$. virgiliae were isolated from more than 10 plant parts. The bud union had significantly higher infection levels compared with the rootstocks but was not different from infection levels found in the pruning wounds and scion (Table 3 ). The infection levels found in the rootstock part of the tree (including pruning wound, bud union, and the rootstock) for the three rootstock cultivars M793 (61.9\%), MM109 (50\%), and G222 (58.8\%) were not significantly different $(P>0.05$; Supplementary Table S3). High infection levels were found in all four nurseries, with percentage of infections ranging from 51.3 to $71.3 \%$ (Table 4 ).

Scion mother block orchards. Of the 310 scion mother block trees, 146 trees $(47 \%)$ were infected with possible canker or woodrotting pathogens. In total, 25 fungal species were identified (Table 2), belonging to seven taxonomic groups. Eutypa lata was isolated from 44 pruning wounds and 26 cankers and was the predominant pathogen isolated in the scion mother block trees. Fungal pathogens isolated from more than 10 pruning wounds or cankers include $T$. versicolor, $D$. seriata, $P$. fraxinopennsylvanicum, $P$. viticola, $D$. rubi-ulmifolii sensu lato, and $P$. minimum. The incidence of infected cankers and pruning wounds was similar, with 27.6 and $27.4 \%$, respectively $(P=0.992)$. The same pathogens were isolated from both the pruning wounds and cankers, with the exception of Didymosphaeria spp. and Diaporthe spp., which were isolated more often from pruning wounds (Supplementary Table S4). Didymosphaeria spp. were isolated from 16 pruning wounds and two cankers found on scion mother block apple trees.

In general, fewer fungal isolates were recovered from cankers found on older trees. The scion mother blocks were divided into three

Table 3. Percent infection in different plant parts isolated from the nursery apple trees

\begin{tabular}{lc}
\hline Plant part & Plant parts infected $(\boldsymbol{\%})^{\mathbf{z}}$ \\
\hline Bud union & $38.40 \mathrm{a}$ \\
Pruning wound & $30.90 \mathrm{ab}$ \\
Scion & $19.70 \mathrm{ab}$ \\
Rootstocks & $15.90 \mathrm{c}$ \\
\hline
\end{tabular}

${ }^{\mathrm{z}}$ Means followed by different letters are significantly different at $P<0.05$.

Table 4. Incidence of nursery apple trees infected with canker and wood rot pathogens sampled from four nurseries

\begin{tabular}{lccc}
\hline & \multicolumn{2}{c}{ Trees } & \\
\cline { 2 - 3 } Nursery & Sampled $(\boldsymbol{n})$ & Infected $(\boldsymbol{n})$ & Infection $(\boldsymbol{\%})$ \\
\hline A & 80 & 41 & 51.30 \\
B & 80 & 57 & 71.30 \\
C & 160 & 112 & 70.00 \\
D & 160 & 100 & 62.50 \\
\hline
\end{tabular}

age groups. Orchards of 10 to 20 years had a significantly higher infection $(66 \%)$ than orchards older than 20 years $(41.4 \%)$. Orchards younger than 10 years old were significantly less infected $(25.2 \%)$ than older trees $(P<0.05)$. Golden Delicious was the most infected cultivar (58.9\%), followed by Early Red One (44.4\%) and, finally, Rosy Glow, with an infection of $25.3 \%$. Infection levels of Early Red One did not differ from Golden Delicious or Rosy Glow; however, the infection in Golden Delicious trees was higher than on Rosy Glow trees $(P=0.007)$. The diversity of fungal taxa isolated from the three different cultivars was similar. E. lata was the predominant fungus isolated from the three cultivars Golden Delicious (41 times), Early Red One (30 times), and Rosy Glow (6 times).

Fruiting bodies of Basidiomycete wood-rotting fungi were present on the dead wood in several of the older mother block orchards. T. versicolor and other wood rot pathogens were isolated from pruning wounds and cankers collected from these orchards. Of the 310 new shoots investigated, $16(5 \%)$ had possible canker and wood rot pathogens present in either the shoot (disks) or the buds. Of the total number of buds isolated, only $0.32 \%$ were infected. The pathogens found in the green shoots were $C$. velutina, E. lata, E. cremea, D. rubi-ulmifolii sensu lato, D. seriata, and T. versicolor (Table 5). These pathogens were also isolated from the cankers and pruning wounds.

Rootstock mother blocks. Layer blocks. Seven fungal species were isolated from asymptomatic layer block shoots (Table 2). All fungal species isolated from the asymptomatic layer block were also found in the nursery trees. The predominant fungal species were $D$. pomorum followed by Truncatella angustata. These two were isolated from both the 1-year-old trees and the nursery trees. The mean percentages of infection of the three rootstock cultivars M793 (23.8\%), MM109 (23\%), and G222 (20.7\%) were not statistically different $(P>0.05)$. Latent infections were detected in 12,24 , and $33 \%$, respectively, of symptomless layer blocks (not statistically different from each other, $P>0.05$ ) from the three PIOs.

One-year-old rootstock trees. Of the 87 symptomatic shoots that were sampled, $36(41 \%)$ shoots harbored possible canker-causing pathogens. For M793, 21 (47\%) shoots and, for MM109, 15 (36\%) shoots harbored possible canker pathogens (data not shown). The predominant pathogen was $D$. seriata, followed by the $C$. schulzeri species complex (Table 2). Both fungal taxa are known dieback pathogens on apple trees. $P$. viticola, $B$. dothidea, and $S$. commune are known dieback pathogens and were also isolated from the nursery material. D. pomorum, which was the predominant species in the asymptomatic material, was reported once from the symptomatic rootstock material. Typical symptoms observed on the affected 1year-old rootstock shoots were constricting cankers. A cross section through the cankers exposed dark-brown vascular discoloration, streaking down into the live tissue of the nonbudded tree. Fruiting structures which formed on the cankerous part of the shoots belonged to $D$. seriata, the $C$. schulzeri species complex, and D. pomorum.

Pathogenicity trial. There were no significant differences between the two trials $(P=0.5563)$; therefore, the trials were combined. For the combined trials, there was a significant species-isolate interaction $(P=0.0001)$. Therefore, the mean lesion lengths and standard deviations for each isolate are presented separately (Table 6). All 72

Table 5. Canker and wood rot pathogens found in the green shoots of the scion mother blocks

\begin{tabular}{|c|c|c|c|c|}
\hline Cultivar & Bud & $\begin{array}{c}\text { Number of buds } \\
\text { infected }\end{array}$ & Disk & $\begin{array}{c}\text { Number of disks } \\
\text { infected }\end{array}$ \\
\hline \multirow[t]{3}{*}{ Golden Delicious } & Trametes versicolor & 1 & $\ldots$ & 0 \\
\hline & Eutypa lata & 1 & $\ldots$ & 0 \\
\hline & Didymosphaeria rubi-ulmifolii sensu lato & 1 & $\ldots$ & 0 \\
\hline \multirow[t]{3}{*}{ Rosy Glow } & $\ldots$ & 0 & D. rubi-ulmifolii sensu lato & 1 \\
\hline & $\ldots$ & 0 & Diplodia seriata & 1 \\
\hline & $\ldots$ & 0 & E. cremea & 1 \\
\hline \multirow[t]{3}{*}{ Early Red One } & D. rubi-ulmifolii sensu lato & 1 & D. seriata & 3 \\
\hline & Coniochaeta velutina & 1 & E. lata & 2 \\
\hline & D. seriata & 3 & $\ldots$ & 0 \\
\hline
\end{tabular}


isolates formed lesions significantly longer than the control (Table 6; Supplementary Figs. 16, 17, 18, and 19). The mean lesion length varied from 15.14 to $95.05 \mathrm{~mm}$. Neofusicoccum viticlavatum isolate STEU 8304 formed the longest lesions $(95.05 \mathrm{~mm})$. However, its lesions were not significantly different from $D$. foeniculina (isolate STEU 8325) and D. eres (B) (isolate STEU 8322). All isolates were reisolated from the discolored tissues of all inoculated shoots, and no inoculated fungi were isolated from the control, fulfilling Koch's postulates. Positive identifications of all species were found when the sequences were compared with the original sequences.

\section{Discussion}

This study found that $65 \%$ of the certified nursery apple trees investigated yielded fungi associated with canker and wood rot symptoms. All nurseries and rootstocks had similar infection rates, suggesting that the problem is not restricted to a specific nursery or rootstock. Canker and wood rot species found from 1-year-old diseased commercial trees were also found in the nursery trees as latent infections.

Forty-five fungal species belonging to 18 genera were identified in this study. Eighteen of these species have been reported on apple trees in South Africa (Adams et al. 2006; Cloete et al. 2011; Crous et al. 2000; Smit et al. 1996, 1997; Sutton et al. 2015); thus, the other
27 species are first reports on apple trees in South Africa. Except for five species, these are also new reports for apple world-wide. Neofusicoccum sp. 4, C. luteo-olivacea, Stereum hirsutum, T. angustata, D. eres (A), and D. eres (B) has been reported from apple trees elsewhere in the world (Farr and Rossman 2019; Slippers et al. 2007; Spadaro et al. 2011; Sutton et al. 2015). Recently, D. eres was divided into two groups A and B (Guarnaccia et al. 2018). Isolates identified as Diaporthe perniciosa fall within D. eres (B). Further taxonomic studies are needed to resolve $D$. eres. The pathogenicity results confirmed that all of the species identified were pathogenic on apple, causing significant lesions on 2-year-old apple shoots.

The five fungal species causing the highest numbers of cankers or dieback in commercial apple orchards were also found at high levels causing latent infections in certified apple nursery trees. These species were $D$. rubi-ulmifolii sensu lato, $D$. seriata, $S$. commune, $D$. pomorum, and C. fasciculata. D. rubi-ulmifolii sensu lato was the dominant species in both certified nursery apple trees and 1-yearold commercial apple trees. These species can be considered the most important species found in this study.

D. rubi-ulmifolii sensu lato, which belongs to the class Dothideomycetes, was the most dominant pathogen causing canker symptoms in commercial orchards and causing latent infections in nursery trees.

Table 6. Mean lesion lengths and percent reisolation of fungal species on field-grown Golden Delicious apple shoots 5 months after inoculation

\begin{tabular}{|c|c|c|c|c|}
\hline \multirow[b]{2}{*}{ Fungal species and isolate number } & \multirow[b]{2}{*}{ Lesion length $(\mathbf{m m})^{x}$} & \multicolumn{2}{|c|}{ Log-transformed mean ${ }^{w}$} & \multirow[b]{2}{*}{ Reisolation $(\%)^{y}$} \\
\hline & & Mean & SD & \\
\hline Neofusicoccum viticlavatum STEU 8304 & $95.05 \mathrm{~A}$ & 4.56 & 0.41 & 67.50 \\
\hline Diaporthe foeniculina STEU 8325 & $77.14 \mathrm{AB}$ & 4.36 & 0.51 & 32.24 \\
\hline D. eres (B) STEU 8322 & $76.93 \mathrm{AB}$ & 4.36 & 0.57 & 75.00 \\
\hline D. eres (A) STEU 8326 & $68.33 \mathrm{BC}$ & 4.24 & 0.48 & 50.63 \\
\hline Neofusicoccum sp. 4 STEU 8284 & $55 \mathrm{CD}$ & 4.03 & 0.51 & 73.13 \\
\hline Diplodia seriata STEU 8299 & $54.55 \mathrm{CD}$ & 4.02 & 0.57 & 73.13 \\
\hline Diaporthe seriata STEU 8300 & $53.36 \mathrm{CDE}$ & 4.00 & 0.38 & 67.50 \\
\hline Phaeoacremonium australiense STEU 8348 & $50.19 \mathrm{DEF}$ & 3.94 & 0.55 & 65.00 \\
\hline P. iranianum STEU 8355 & $46.35 \mathrm{D}-\mathrm{G}$ & 3.86 & 0.29 & 80.63 \\
\hline P. subulatum STEU 8362 & $45.97 \mathrm{D}-\mathrm{G}$ & 3.85 & 0.55 & 55.63 \\
\hline D. eres (A) STEU 8327 & $45.42 \mathrm{D}-\mathrm{H}$ & 3.84 & 0.50 & 39.38 \\
\hline P. viticola STEU 8364 & $45.39 \mathrm{D}-\mathrm{H}$ & 3.84 & 0.44 & 39.38 \\
\hline Coniochaeta fasciculata STEU 8312 & $44.73 \mathrm{D}-\mathrm{H}$ & 3.82 & 0.51 & 6.88 \\
\hline D. ambigua STEU 8320 & $44.51 \mathrm{D}-\mathrm{H}$ & 3.82 & 0.53 & 30.00 \\
\hline P. prunicola STEU 8359 & $43.77 \mathrm{D}-\mathrm{I}$ & 3.80 & 0.58 & 45.63 \\
\hline P. longicollarum STEU 8402 & $43.11 \mathrm{D}-\mathrm{J}$ & 3.79 & 0.48 & 34.38 \\
\hline D. cynaroidis STEU 8321 & $42.34 \mathrm{D}-\mathrm{K}$ & 3.77 & 0.65 & 28.95 \\
\hline D. eres (B) STEU 8322 & $42.22 \mathrm{D}-\mathrm{K}$ & 3.77 & 0.62 & 56.25 \\
\hline P. inflatipes STEU 8353 & $41.45 \mathrm{D}-\mathrm{L}$ & 3.75 & 0.37 & 71.25 \\
\hline P. prunicola STEU 8358 & $41.41 \mathrm{D}-\mathrm{L}$ & 3.75 & 0.5 & 56.25 \\
\hline P. austroafricanum STEU 8349 & $41.35 \mathrm{D}-\mathrm{L}$ & 3.75 & 0.42 & 56.88 \\
\hline $\begin{array}{l}\text { Didymosphaeria rubi-ulmifolii sensu lato } \\
\text { STEU } 8404\end{array}$ & $41.23 \mathrm{D}-\mathrm{L}$ & 3.74 & 0.44 & 35.00 \\
\hline P. viticola STEU 8363 & $40.64 \mathrm{D}-\mathrm{M}$ & 3.73 & 0.47 & 61.88 \\
\hline$P$. geminum STEU 8400 & $40.03 \mathrm{E}-\mathrm{N}$ & 3.71 & 0.47 & 65.63 \\
\hline P. austroafricanum STEU 8350 & $39.67 \mathrm{E}-\mathrm{O}$ & 3.71 & 0.60 & 61.88 \\
\hline P. subulatum STEU 8361 & $39.56 \mathrm{E}-\mathrm{O}$ & 3.70 & 0.45 & 50.00 \\
\hline P. scolyti STEU 8360 & $39.55 \mathrm{E}-\mathrm{O}$ & 3.70 & 0.51 & 51.25 \\
\hline D. rubi-ulmifolii sensu lato STEU 8405 & $38.92 \mathrm{~F}-\mathrm{P}$ & 3.69 & 0.53 & 47.5 \\
\hline Cadophora luteo-olivacea STEU 8308 & $38.75 \mathrm{~F}-\mathrm{P}$ & 3.68 & 0.59 & 74.38 \\
\hline Didymella pomorum STEU 8339 & $38.21 \mathrm{~F}-\mathrm{Q}$ & 3.67 & 0.43 & 36.88 \\
\hline Coniochaeta fasciculata STEU 8313 & $38.21 \mathrm{~F}-\mathrm{Q}$ & 3.67 & 0.51 & 21.71 \\
\hline P. australiense STEU 8347 & $37.03 \mathrm{G}-\mathrm{R}$ & 3.64 & 0.5 & 73.13 \\
\hline P. gетіпит STEU 8401 & $36.78 \mathrm{G}-\mathrm{R}$ & 3.63 & 0.52 & 56.25 \\
\hline Coniochaeta velutina STEU 8314 & $36.69 \mathrm{G}-\mathrm{R}$ & 3.63 & 0.59 & 27.50 \\
\hline C. velutina STEU 8315 & $36.56 \mathrm{G}-\mathrm{R}$ & 3.63 & 0.48 & 27.50 \\
\hline \multirow[t]{2}{*}{ Truncatella angustata STEU 8284} & $36.34 \mathrm{G}-\mathrm{R}$ & 3.62 & 0.56 & 45.00 \\
\hline & & & & (Continued on next page) \\
\hline
\end{tabular}

${ }^{\mathrm{w}}$ Least significant difference of logarithmic transformed lesion lengths $=0.2969 . \mathrm{SD}=$ standard deviation.

x Back-transformed mean lesion length is based on 20 replicates, with 10 replicates per orchard. Means followed by different letters differ significantly $(P<0.05)$ according to Student's $t$ test.

y Mean reisolation.

z $\mathrm{PDA}+=$ potato dextrose agar amended with streptomycin sulfate. 
Members of the class Dothideomycetes are lesser known as cankercausing pathogens. However, a pathogenicity study conducted by Cloete et al. (2011) found that D. rubi-ulmifolii sensu lato, previously known as Paraconiothyrium brasiliense Verkley, formed significant lesions on apple shoots. Cloete et al. (2011) only isolated the pathogen from canker symptoms collected from diseased pear trees. Recently, D. rubi-ulmifolii sensu lato was found to be the causal agent of cankers identified near the base of young apple trees that resulted in a loss of 13,928 trees (57\%) from 2012 until 2018 in Ethiopia (A. Yirgu and A. Gezahgne, the Central Ethiopia Environment and Forest Research Center, personal communication). These losses were incurred by one producer who decided to remove their apple orchards in March 2019 due to the high occurrence of cankers. Little is known regarding the biology of $D$. rubi-ulmifolii sensu lato; therefore this occurrence requires further investigation.

Even though infection levels in the nursery trees were high, apple producers do not find a similarly high disease incidence of cankers or wood rot in newly established commercial orchards. Canker and wood rot pathogens are opportunistic and can induce symptoms when the host experiences stress (Menapace et al. 2015). Symptoms of the canker pathogen Valsa mali var. mali were observed due to stress experienced by the apple trees (Zang et al. 2012). In the present study, one soil sample for each of the 13 orchards was analyzed for physiochemical characteristics (data not shown). In general, the soils with a high phosphorus level indicated that the plant had difficulty taking up water and a low, acidic $\mathrm{pH}$ indicated an aluminum toxicity (Jensen 2010; Marschner et al. 1987). These factors indicate that the trees were planted in suboptimal soil, which will place either toxicity or mineral deficiency stress on the trees (Jensen 2010; Marschner et al. 1987). These findings need to be confirmed with more soil samples per orchard analyzed. In general, stress conditions contribute to a more vulnerable tree for the development of dieback.

The presence of the wood rot pathogens in both the young trees and the nursery trees was surprising because these pathogens usually occur on older trees and dead wood (Matthee and Thomas 1977). The fruiting bodies of Ascomycete and Basidiomycete fungi were found on the diseased or dead 1-year-old apple trees. These fruiting bodies produce spores, contributing to the aerial inoculum present in orchards. The spores can be spread with wind and rain and potentially infect new wounds (Matthee and Thomas 1977; Valiuškaite and Raudonis 2008). For this reason, it is very important to remove the dead trees from the young orchards.

Most of the cankers formed on the scion shoot of the 1-year-old commercial apple trees. McCracken et al. (2003) also found that cankers caused by $N$. ditissima mostly developed on the scion shoots after establishing apple trees. Infection of the scion shoot can occur during the final stages of propagation when the lateral shoots are removed from the main stem. Alternatively, infections can also occur through wounds made on the scion shoot in newly established orchards.

Table 6. (Continued from previous page)

\begin{tabular}{|c|c|c|c|c|}
\hline \multirow[b]{2}{*}{ Fungal species and isolate number } & \multirow[b]{2}{*}{ Lesion length $(\mathbf{m m})^{x}$} & \multicolumn{2}{|c|}{ Log-transformed mean ${ }^{w}$} & \multirow[b]{2}{*}{ Reisolation $(\%)^{\mathrm{y}}$} \\
\hline & & Mean & $\overline{\text { SD }}$ & \\
\hline $\begin{array}{l}\text { Cytospora schulzeri species complex STEU } \\
8317\end{array}$ & $36.25 \mathrm{G}-\mathrm{R}$ & 3.62 & 0.63 & 8.13 \\
\hline Cadophora luteo-olivacea STEU 8309 & $35.3 \mathrm{G}-\mathrm{S}$ & 3.59 & 0.56 & 62.50 \\
\hline Cadophora sp. STEU 8311 & $35.1 \mathrm{G}-\mathrm{S}$ & 3.59 & 0.65 & 68.13 \\
\hline Coniochaeta sp. STEU 8316 & $34.96 \mathrm{G}-\mathrm{T}$ & 3.58 & 0.57 & 16.88 \\
\hline Cadophora sp. STEU 8310 & $34.7 \mathrm{G}-\mathrm{T}$ & 3.58 & 0.49 & 48.75 \\
\hline Eutypella citricola STEU 8337 & $33.85 \mathrm{H}-\mathrm{U}$ & 3.55 & 0.58 & 28.75 \\
\hline Didymosphaeria variabile STEU 8344 & $33.78 \mathrm{H}-\mathrm{U}$ & 3.55 & 0.37 & 49.38 \\
\hline Eutypella citricola STEU 8338 & $32.68 \mathrm{I}-\mathrm{V}$ & 3.52 & 0.61 & 16.25 \\
\hline Didymella pomorum STEU 8341 & $32.67 \mathrm{I}-\mathrm{V}$ & 3.52 & 0.50 & 36.88 \\
\hline Didymosphaeria variabile STEU 8343 & $32.45 \mathrm{I}-\mathrm{W}$ & 3.51 & 0.49 & 65.00 \\
\hline Bjerkandera adusta STEU 8285 & $31.94 \mathrm{~J}-\mathrm{W}$ & 3.49 & 0.60 & 30.63 \\
\hline Truncatella angustata STEU 8283 & $31.76 \mathrm{~K}-\mathrm{W}$ & 3.49 & 0.41 & 43.13 \\
\hline Peniophora sp. 1 STEU 8288 & $31.28 \mathrm{~K}-\mathrm{X}$ & 3.47 & 0.46 & 1.88 \\
\hline Trametes versicolor STEU 8296 & $30.89 \mathrm{~L}-\mathrm{X}$ & 3.46 & 0.48 & 31.25 \\
\hline Diaporthe virgiliae STEU 8328 & $30.68 \mathrm{~L}-\mathrm{X}$ & 3.46 & 0.44 & 34.38 \\
\hline Didymella pomorum STEU 8340 & $30.6 \mathrm{~L}-\mathrm{X}$ & 3.45 & 0.51 & 21.88 \\
\hline $\begin{array}{l}\text { Didymosphaeria rubi-ulmifolii sensu lato } \\
\text { STEU } 8403\end{array}$ & $30.36 \mathrm{M}-\mathrm{X}$ & 3.45 & 0.44 & 50.63 \\
\hline Didymella pomorum STEU 8342 & $30.14 \mathrm{M}-\mathrm{X}$ & 3.44 & 0.45 & 26.25 \\
\hline Diaporthe ambigua STEU 8319 & $30.05 \mathrm{M}-\mathrm{X}$ & 3.44 & 0.46 & 44.38 \\
\hline $\begin{array}{l}\text { Cytospora schulzeri species complex STEU } \\
8318\end{array}$ & $29.93 \mathrm{~N}-\mathrm{X}$ & 3.43 & 0.61 & 10.00 \\
\hline Eutypa cremea STEU 8335 & $29.41 \mathrm{O}-\mathrm{Y}$ & 3.41 & 0.47 & 32.5 \\
\hline D. amygdali STEU 8330 & $28.81 \mathrm{P}-\mathrm{Y}$ & 3.39 & 0.44 & 58.75 \\
\hline E. cremea STEU 8336 & $28.67 \mathrm{P}-\mathrm{Y}$ & 3.39 & 0.50 & 22.50 \\
\hline Bjerkandera adusta STEU 8286 & $28.47 \mathrm{Q}-\mathrm{Y}$ & 3.38 & 0.64 & 25.00 \\
\hline Trametes versicolor STEU 8295 & $28.07 \mathrm{R}-\mathrm{Y}$ & 3.37 & 0.47 & 28.75 \\
\hline $\begin{array}{l}\text { Didymosphaeria rubi-ulmifolii sensu lato } \\
\text { STEU } 8346\end{array}$ & $27.95 \mathrm{R}-\mathrm{Y}$ & 3.37 & 0.48 & 48.13 \\
\hline D. rubi-ulmifolii sensu lato STEU 8345 & $26.44 \mathrm{~S}-\mathrm{Z}$ & 3.31 & 0.56 & 16.25 \\
\hline Peniophora sp. 2 STEU 8291 & $25.81 \mathrm{~T}-\mathrm{Z}$ & 3.29 & 0.45 & 23.75 \\
\hline Botryosphaeria dothidea STEU 8298 & $25.22 \mathrm{U}-\mathrm{Z}$ & 3.27 & 0.50 & 79.61 \\
\hline Diaporthe foeniculina STEU 8324 & $24.6 \mathrm{~V}-\mathrm{Z}$ & 3.24 & 0.58 & 54.38 \\
\hline D. amygdali STEU 8331 & $23.86 \mathrm{~W}-\mathrm{Z}$ & 3.21 & 0.41 & 56.25 \\
\hline Peniophora sp.1 STEU 8289 & $23.03 \mathrm{XYZ}$ & 3.18 & 0.59 & 26.25 \\
\hline Stereum hirsutum STEU 8294 & $21.77 \mathrm{YZ}$ & 3.13 & 0.49 & 17.50 \\
\hline Botryosphaeria dothidea STEU 8297 & $21.71 \mathrm{YZ}$ & 3.12 & 0.56 & 70.63 \\
\hline D. virgiliae STEU 8329 & $20.05 \mathrm{aZ}$ & 3.05 & 0.62 & 53.75 \\
\hline Peniophora sp. 2 STEU 8290 & $15.14 \mathrm{a}$ & 2.78 & 0.43 & 21.71 \\
\hline Control $(\mathrm{PDA}+)^{\mathrm{z}}$ & $8.68 \mathrm{~b}$ & 2.27 & 0.49 & 0 \\
\hline
\end{tabular}


The pruning wound on the rootstock and the bud union had the highest infection in the nursery trees. Canker and wood rot symptoms that developed from these sites were found in $19 \%$ of the infected 1-year-old apple trees. These two wound sites are important sites for infection. Infection occurs via aerial inoculum present in the nursery when the wounds are made or via infected bud material. Older apple orchards and other fruit hosts in close vicinity can harbor similar pathogens and can contribute to the aerial inoculum present (Damm et al. 2008; Halleen et al. 2007; Slippers et al. 2007).

Canker and wood rot fungi isolated from pruning wounds and cankers from scion mother block trees were similar to those found in 1year-old apple trees and nursery trees. E. lata was the predominant species found in the scion mother block cultivars; however, it was seldom found in the young nursery trees. This pathogen often occurs on grapevines causing Eutypa dieback (Trouillas and Gubler 2010) and is commonly associated with decline on older vines (Moyo et al. 2016; White et al. 2011). Cloete et al. (2011) also isolated this pathogen from older apple orchards, which can suggest that $E$. lata prefers older tissue and is why this pathogen was not commonly found in young nursery trees.

Golden Delicious trees had higher infection levels than Rosy Glow trees. This is probably due to the average ages of the orchards. Rosy Glow is a newer cultivar, with an average age of 8 years, compared with Golden Delicious orchards, which had an average age of 20 years. The younger orchards, which in this case were all Rosy Glow trees, had few to no cankers on the trees. It is expected that older orchards would harbor more canker pathogens because they have been pruned for longer, accumulating more wounds on the trees, which have been exposed to aerial inoculum for longer. High infection levels in the pruning wounds indicate that apple wounds are susceptible to infection by canker and wood rot pathogens that may result in the development of cankers. In some of the older, severely diseased trees with larger cankers, the causal organisms were not isolated easily, resulting in a lower infection percentage obtained for trees older than 20 years versus between 10 and 20 years in age. This can be due to the hardiness of the wood after several years, or the pathogen may only be present as fruiting structures on the surface of the canker.

A low infection rate $(5 \%)$ was found in the new green shoots, however; this study shows that infection of newly grafted plants can occur directly through infected scion buds used during propagation. The fungal pathogens D. rubi-ulmifolii sensu lato, E. lata, and T. versicolor were isolated from infected scion buds and were also found in the nursery trees and 1-year-old diseased orchards.

The rooted rootstock cuttings also proved to be a direct inoculum source for infection of the nursery trees, with an overall infection of $21 \%$. The predominant pathogens found were D. pomorum, which have been reported as canker-causing pathogens of apple in South Africa by Crous et al. (2000). This was followed by T. angustata, which is found as a weaker pathogen that can cause decline of grapevines in Iran (Arzanlou et al. 2013), Texas (United States) (Úrbez-Torres et al. 2009), and Australia (Sergeeva et al. 2005). Latent infections of the rootstock layer blocks by $D$. pomorum, $T$. angustata, $B$. dothidea, and $D$. foeniculina were also found in the nursery trees. This latent infection in the rootstock layer blocks can be expressed when shoots experience stress similar to what was seen in the 1-year-old nursery rootstock blocks. Fruiting structures were found on the cankers of the 1-year-old nursery shoots that can provide aerial inoculum during budding.

The incidence of dieback in 1-year-old commercial orchards due to canker and wood rot can be reduced by managing abiotic and biotic stress factors placed on the apple tree. This should be done throughout the propagation process, during storage, and during the establishment of young trees. Abiotic stresses responsible for low productivity in apple orchards include fluctuation in temperature, nutrient deficiencies and toxicities, low-temperature injuries such as frost, water logging, insect damage, and improper horticultural practices (Arzanlou and Bakhshi 2012; Krishna et al. 2010; Marek et al. 2013; Slippers et al. 2007; Valiuškaitè and Raudonis 2008).
To reduce infection levels in nursery trees, aerial inoculum in the nurseries as well as in commercial orchards can be reduced by applying sanitation practices. Sanitation practices include the removal of dead and cankered material, especially when fruiting bodies are present. The importance of sanitation has been shown for the management of E. lata and Valsa leucostoma in stone fruit orchards (Bertrand and English 1976; Carter 1983). Sanitation is important in all phases of propagation as well as in established orchards. When the canker forms on the scion shoot of a 1-year-old tree, the diseased part of the tree can be removed. The tree will produce a new scion shoot in the next growing season; this will allow the tree to recover from the infection.

The cankered rooted rootstocks in the 1-year-old nursery blocks should be removed before budding as well as before the rootstock shoot is pruned back. This will reduce infection during budding. A pruning wound protectant containing a fungicide should be used throughout the propagation process to reduce infections in nurseries. Younger scion orchards were less infected than older orchards; thus, PIOs should renew mother block orchards at a younger age. This will reduce the infection of the scion buds used during propagation.

Certified nursery apple trees were infected with canker and wood rot pathogens, which resulted in the distribution of seemingly healthy trees to farmers. Nursery trees get infected during the propagation process via aerial inoculum, which is present at the time of budding and pruning back. The plant material used during propagation also plays a role in infecting the nursery apple trees because a direct infection was found via the scion bud and rooted rootstock cutting. The young trees expressed canker and wood rot symptoms shortly after experiencing stress conditions during the establishment of the orchards. The postnursery handling of nursery trees is very important; to what extent storage conditions of trees and optimal planting and postplanting practices would contribute to the expression of latent infections of canker and wood rot pathogens needs to be further investigated. Future work should aim to lessen infections via propagation material and provide efficient protection of the pruning wound.

\section{Literature Cited}

Adams, G. C., Roux, J., and Wingfield, M. J. 2006. Cytospora species (Ascomycota, Diaporthales, Valsaceae): Introduced and native pathogens of trees in South Africa. Australas. Plant Pathol. 35:521-548.

Arzanlou, M., and Bakhshi, M. 2012. ITS-rDNA sequences differentiate a new lineage of Diplodia associated with canker disease of apple in Iran. Plant Pathol. Quar. 2:132-141.

Arzanlou, M., Narmani, A., Moshari, S., Khodaei, S., and Babai-Ahari, A. 2013. Truncatella angustata associated with grapevine trunk disease in Northern Iran. Arch Phytopathol. Plant Prot. 46:1168-1181.

Bertrand, P. F., and English, H. 1976. Release and dispersal of conidia and ascospores of Valsa leucostoma. Phytopathology 66:987-991.

Borovinova, M., Petrova, V., and Maneva, S. 2012. Effect of different growing systems of apple on trunk and branch diseases and pests. Not. Bot. Horti Agrobot. Cluj-Na 40:159-162.

Brown, A. E., Muthumeenakashi, S., Swinburne, T. R., and Li, R. 1994. Detection of the source of the infection of apple trees by Cylindrocarpon heteronema using DNA polymorphisms. Plant Pathol. 43:338-343.

Brown-Rytlewski, D. E., and McManus, P. S. 2000. Virulence of Botryosphaeria dothidea and Botryosphaeria obtusa on apple and management of stem cankers with fungicides. Plant Dis. 84:1031-1037.

Carbone, I., and Kohn, L. M. 1999. A method for designing primer sets for speciation studies in filamentous ascomycetes. Mycologia 91:553-556.

Carter, M. V. 1983. Biological control of Eutypa armeniacae 5. Guidelines for establishing routine wound protection in commercial apricot orchards. Aust. J. Exp. Agric. Anim. Husb. 23:429-436.

Cloete, M., Fourie, P. H., Damm, U., Crous, P. W., and Mostert, L. 2011. Fungi associated with die-back symptoms of apple and pear trees, a possible inoculum source of grapevine trunk disease pathogens. Phytopathol. Mediterr. 50:176-190.

Crous, P. W., Phillips, A. J. L., and Baxter, A. P. 2000. Phytopathogenic Fungi from South Africa, 1st ed. Department of Plant Pathology Press, Stellenbosch University, Stellenbosch, South Africa.

Damm, U., Mostert, L., Crous, P. W., and Fourie, P. H. 2008. Novel Phaeoacremonium species associated with necrotic wood of Prunus trees. Persoonia 20:87-102.

Elfar, K., Torres, R., Díaz, G. A., and Latorre, B. A. 2013. Characterization of Diaporthe australafricana and Diaporthe spp. associated with stem canker on blueberry in Chile. Plant Dis. 97:1042-1050. 
Farr, D. F., and Rossman, A. Y. 2019. Fungal Databases, U.S. National Fungus Collections. United States Department of Agriculture-Agricultural Research Service. https://nt.ars-grin.gov/fungaldatabases/

Fujita, K., Sugiki, T., and Matsunaka, K. 1988. Apple blight caused by Diaporthe tanakae in Aomori prefecture. (Abstr.) Bull. Aomori Field Crop Hortic. Exp. Stn. 6:17-35.

Glass, N., and Donaldson, G. 1995. Development of primer sets designed for use with the PCR to amplify conserved genes from filamentous ascomycetes. Appl. Environ. Biol. 16:1323-1330.

Guarnaccia, V., Groenewald, J. Z., Woodhall, J., Armengol, J., Cinelli, T., Eichmeier, A., Ezra, D., Fontaine, F., Gramaje, D., Gutierrez-Aguirregabiria, A., Kaliterna, J., Kiss, L., Larignon, P., Luque, J., Mugnai, L., Naor, V., Raposo, R., Sándor, E., Váczy, K. Z., and Crous, P. W. 2018. Diaporthe diversity and pathogenicity revealed from a broad survey of grapevine diseases in Europe. Persoonia 40:135-153.

Guindon, S., Dufayard, J. F., Lefort, V., Anisimova, M., Hordijk, W., and Gascuel, O. 2010. New algorithms and methods to estimate maximum-likelihood phylogenies: Assessing the performance of PhyML 3.0. Syst. Biol. 59:307-321.

Guindon, S., and Gascuael, O. 2003. A simple, fast, and accurate algorithm to estimate large phylogenies by maximum likelihood. Syst. Biol. 52:696-704.

Halleen, F., Mostert, L., and Crous, P. W. 2007. Pathogenicity testing of lesserknown vascular fungi of grapevines. Australas. Plant Pathol. 36:277-285.

Hillis, D. M., and Bull, J. J. 1993. An empirical test of bootstrapping as a method for assessing confidence in phylogenetic analysis. Syst. Biol. 42:182-192.

Jensen, T. L. 2010. Soil $\mathrm{pH}$ and the availability of plant nutrients. International Plant Nutrition Institute, Norcross, GA, U.S.A. http://www.ipni.net/ publication/pnt-na.nsf/0/013f96e7280a696985257cd6006fb98f

John, J. A., and Quenouille, M. H. 1977. Experiments; Design and Analysis. Charles Griffin \& Co. Ltd., London, High Wycombe, United Kingdom.

Katoh, K., Misawa, K., Kuma, K., and Miyata, T. 2002. MAFFT: A novel method for rapid multiple sequences alignment based on fast Fourier transform. Nucleic Acids Res. 30:3059-3066.

Katoh, K., and Standley, D. M. 2013. MAFFT multiple sequence alignment software version 7: Improvements in performance and usability. Mol. Biol. Evol. 30:772-780.

Krishna, H., Das, B., Attri, B. L., Grover, M., and Ahmed, N. 2010. Suppression of Botryosphaeria canker of apple by arbuscular mycorrhizal fungi. Crop Prot. 29: 1049-1054.

Levene, H. 1960. Robust test in the equality of variance. Pages 278-292 in: Contributions to Probability and Statistics: Essays in Honor of Harold Hotelling. I. Olkin, ed. Stanford University Press, Stanford, CA, U.S.A.

Marek, S. M., Yaghmour, M. A., and Bostock, R. M. 2013. Fusarium spp., Cylindrocarpon spp., and environmental stress in the etiology of a canker disease of cold-stored fruit and nut tree seedlings in California. Plant Dis. 97: 259-270.

Marschner, H., Römheld, V., and Cakmak, I. 1987. Root-induced changes of nutrient availability in the rhizosphere. J. Plant Nutr. 10:1175-1184.

Martín, M. T., Cuesta, M. J., and Martín, L. 2014. Development of SCAR primers for PCR assay to detect Diplodia seriata. Int. Scholarly Res. Not. 2014: Article 824106.

Matthee, F. N., and Thomas, A. C. 1977. Wood-rotting fungi of fruit trees and vines. I. Diagnosing the main pathogens. Deciduous Fruit Grow. July.

McCracken, A. R., Berrie, A., Barbara, D. J., Locke, T., Cooke, L. R., Phelps, K., Swinburne, T. R., Brown, A. E., Ellerker, B., and Langrell, S. R. H. 2003. Relative significance of nursery infection and orchard inoculum in the development and spread of apple canker (Nectria galligena) in young orchards. Plant Pathol. 52:553-566.

Menapace, L., Colson, G., and Raffaelli, R. 2015. Climate change beliefs and perceptions of agricultural risks: An application of the exchangeability method. Glob. Environ. Change 35:70-81.

Moyo, P., Mostert, L., Bester, M., and Halleen, F. 2016. Trunk disease fungi associated with Diospyros kaki in South Africa. Plant Dis. 100:2383-2393.

O’Donnell, K., and Cigelnik, E. 1997. Two divergent intragenomic rDNA ITS2 types within a monphylic lineage of the fungus Fusarium are nonorthologous. Mol. Phylogenet. Evol. 7:103-116.

Ott, R. L., and Longnecker, M. 2001. An Introduction to Statistical Methods and Data Analysis, 5th ed. Duxbury Press, Belmont, CA, U.S.A.

Sergeeva, V., Priest, M., and Nair, N. G. 2005. Species of Pestaliopsis and related genera occurring on grapevines in Australia. Australas. Plant Pathol. 34: 255-258.

Shapiro, S. S., and Wilk, M. B. 1965. An analysis of variance test for normality (complete samples). Biometrika 52:591-611.

Slippers, B., Smit, B. A., Crous, P. W., Coutinho, T. A., Wingfield, B. D., and Wingfield, M. J. 2007. Taxonomy, phylogeny and identification of Botryosphaeriaceae associated with pome and stone fruit trees in South Africa and other regions of the world. Plant Pathol. 56:128-139.

Smit, W. A., Viljoen, C. D., Wingfield, B. D., Wingfield, M. J., and Calitz, F. J. 1996. A new canker disease of apple, pear and plum rootstocks caused by Diaporthe ambigua in South Africa. Plant Dis. 80:1331-1335.

Smit, W. A., Wingfield, B. D., and Wingfield, M. J. 1997. Vegetative incompatibility in Diaporthe ambiqua. Plant Pathol. 46:366-372.

Snedecor, G. W., and Cochran, W. G. 1980. Statistical Methods, 7th ed. Iowa State University Press, Ames, IA, U.S.A.

Spadaro, D., Pellegrino, C., Garibaldi, A., and Gullino, M. L. 2011. Development of SCAR primers for the detection of Cadophora luteo-olivacea on kiwifruit and pome fruit and of Cadophora malorum on pome fruit. Phytopathol. Mediterr. 50:430-441.

Sutton, T. B., Aldwinckle, H. S., Agnello, A. M., and Walgenbach, J. F. 2015. Canker and wood rot diseases. Pages 48-62 in: Compendium of Apple and Pear Diseases and Pests. American Phytopathological Society, St. Paul, MN, U.S.A.

Trouillas, F. P., and Gubler, W. D. 2010. Host range, biological variation, and phylogenetic diversity of Eutypa lata in California. Phytopathology 100:1048-1056.

Úrbez-Torres, J. R., Adams, P., Kamas, J., and Gubler, W. D. 2009. Identification, incidence, and pathogenicity of fungal species associated with grapevine dieback in Texas. Am. J. Enol. Vitic. 60:497-507.

Valiuškaitè, A., and Raudonis, L. 2008. Epidemiology of bark diseases of apple tree in Lithuania. Sci. Works Lith. Inst. Hortic. Lith. Univ. Agric. 27:51-57.

White, C., Halleen, F., and Mostert, L. 2011. Symptoms and fungi associated with esca in South African vineyards. Phytopathol. Mediterr. 50:236-246.

White, T. J., Bruns, T., Lee, S., and Taylor, J. 1990. Amplification and direct sequencing of fungal ribosomal RNA genes for phylogenetics. Pages 315-322 in: PCR Protocols: A Guide to Methods and Applications. M. A Innis, D. H. Gelfand, and J. J. Sninsky, eds. Academic Press Inc., New York, NY, U.S.A.

Zang, R., Kang, Z., and Huang, L. 2012. A nested PCR assay for detecting Valsa mali var. mali in different tissues of apple trees. Plant Dis. 96:1645-1652.

Zhang, Q., Wang, C., Yong, D., Li, G., Dong, X., and Li, B. 2014. Induction of resistance mediated by an attenuated strain of Valsa mali var. mali using pathogen-apple callus interaction system. Sci. World J. 2014: Article 201382 . 